\title{
Some Existence Results for Fully Nonlinear Elliptic Equations of Monge-Ampère Type
}

\author{
YANYAN LI \\ Courant Institute
}

\begin{abstract}
We study the existence and uniqueness of solutions of Monge-Ampère-type equations. This type of equations has been studied extensively by Caffarelli, Nirenberg, Spruck and many others. (See [5] through [8] and the references therein.) We present some existence and uniqueness results for this type of equations on compact Riemannian manifolds with non-negative sectional curvature. We have also generalized some results in [7].
\end{abstract}

\section{Introduction}

Recently there has been an enormous development in the existence theory of nonlinear elliptic equations; see [5],[6], [7], [8] and the references there. Here we give some additional results on the existence and uniqueness of solutions of the same type of equations.

Throughout this paper $f$ will be a smooth function defined in an open convex cone $\Gamma \subset \mathbf{R}^{n}$, which is different from $\mathbf{R}^{n}$, with vertex at the origin, containing the positive cone: $\left\{\lambda \in R^{n}\right.$ : each component $\left.\lambda_{i}>0\right\}$ and satisfying the following in $\Gamma$ :

$$
\lim _{\substack{\lambda \rightarrow 0 \\ \lambda \in \Gamma}} \inf f(\lambda)>-\infty
$$

$$
\frac{\partial f}{\partial \lambda_{i}}>0 \text { for all } i
$$

$$
f \text { is a concave function. }
$$

$\Gamma$ and $f$ are assumed to be invariant under interchange of any two $\lambda_{i}$, namely, they are symmetric in $\lambda_{i}$. It follows that

$$
\Gamma \subset\left\{\sum \lambda_{i}>0\right\}
$$

In each of the sections below, $f$ is required to satisfy further conditions, which may be different, but are always satisfied by the symmetric functions if not 
noted otherwise:

$$
f(\lambda)=\left(\sum_{i_{1}<\cdots<i_{n}} \lambda_{i_{1}} \cdots \lambda_{i_{k}}\right)^{1 / k},
$$

with $\Gamma$ the connected component of $\left\{\lambda \in \mathbb{R}^{\prime \prime}: \sum_{i_{1}<\cdots<i_{k}} \lambda_{i_{1}} \cdots \lambda_{i_{k}}>0\right\}$ containing $\left\{\lambda \in \mathbb{R}^{n}: \lambda_{i}>0\right\}$. As to the properties of $f(\lambda)=\left(\sum_{i_{1}}<\right.$ $\left.\cdots<i_{k} \lambda_{i_{1}} \cdots \lambda_{i_{k}}\right)^{1 / k}$, see [7] and the Appendix at the end of this paper.

In Section 1, we give some existence and uniqueness results for certain fully nonlinear elliptic equations on compact Riemannian manifolds with non-negative sectional curvature. We make use of the degree theory for second-order fully nonlinear-elliptic operators introduced in [11] and [18] to prove the existence results and therefore must make a priori estimates for solutions of the equation. Due to the results of Evans and Krylov, we only need to estimate derivatives up to second order; see [10], [17]. Further results on a flat torus are given in the second section and, in Sections 3 and 4, we generalize some results of Caffarelli, Nirenberg and Spruck in [7].

\section{Existence and Uniqueness of Certain Fully Nonlinear Elliptic Equations on Compact Riemannian Manifolds with Non-negative Sectional Curvature}

In this section, $(M, g)$ is a smooth compact $n$-dimensional Riemannian manifold with non-negative sectional curvature and $f$ satisfies (1), (2), and the following condition:

$$
\lim _{\substack{|\lambda| \rightarrow+\infty \\ \lambda \in \Gamma \\ f(\lambda) \geq \delta}} \sum_{i} f_{\lambda_{1}}(\lambda)=+\infty \text { for all } \delta>0
$$

Notice that condition (5) is satisfied by the symmetric functions (4) only for $k \geqq 2$; however, in the case $k=1$ the principle part of the differential operator is in fact the Laplace operator, everything is clear then.

For $u \in C^{2}(M)$ we denote the Hessian of $u$ by $H(u)$; namely,

$$
H(u)(X, Y)=X Y u-\nabla_{X} Y u
$$

where $X, Y$ are tangent vectors, $\nabla_{X} Y$ is the covariant derivative of $Y$ along the direction $X$; see [3]. It is well known that $H(u)$ is symmetric:

$$
H(u)(X, Y)=H(u)(Y, X)
$$

Consider the following equation:

$$
f(\lambda(\mathrm{Id}+H(u)))=\psi(x, u)
$$


where $\lambda(\operatorname{Id}+H(u))=\left(\lambda_{1}, \cdots, \lambda_{n}\right)$ are eigenvalues of $\operatorname{Id}+H(u)$ and $\psi$ satisfies the following hypotheses:

There exist two constants $a_{1}<A_{1}$, such that

$$
\max _{x \in M} \psi\left(x, a_{1}\right) \leqq f(1, \cdots, 1) \leqq \min _{x \in M} \psi\left(x, A_{1}\right) .
$$

There exists a positive constant $\delta_{0}>0$, such that

$$
\begin{aligned}
& \qquad \psi(x, s)>\delta_{0}>\lim _{\lambda \rightarrow \lambda_{0}} \sup f(\lambda) \\
& \text { for all } \lambda_{0} \in \partial \Gamma, \quad x \in M, \quad a_{1} \leqq s \leqq A_{1} .
\end{aligned}
$$

We now state the main result of this section.

THEOREM 1.1. Let $(M, g)$ be a smooth compact n-dimensional Riemannian manifold with non-negative sectional curvature. Suppose that $f, \psi$ satisfy (1), (2), (5), (7), and (8); then (6) has at least one solution $u \in C^{\infty}(M)$ with $a_{1} \leqq u \leqq A_{1}$, $\lambda($ Id $+H(u)) \in \Gamma$. If $\psi_{u} \geqq 0$ for all $x \in M, a_{1} \leqq u \leqq A_{1}$, such $a$ solution is unique up to constants.

The uniqueness part follows from the following simple argument:

Let $u, v$ be two solutions of (6); then

$$
f(\lambda(\text { Id }+H(u)))-f(\lambda(\text { Id }+H(v)))-\psi(x, u)+\psi(x, v)=0,
$$

$u-v$ satisfies an elliptic equation for which the maximum principle holds $\left(\psi_{u} \geqq 0\right)$. Therefore, $u-v \equiv$ constant.

We shall prove the existence of at least one solution by using the degree theory for second-order fully nonlinear elliptic operators introduced in [18]. First we need to make a priori estimates for the solutions of (6).

The $C^{0}$ estimate follows from our hypotheses and the maximum principle. We obtain the $C^{1}$ and $C^{2}$ estimates by choosing suitable test functions. The $C^{2 . \alpha}$ estimates follow from the $C^{2}$ estimates and the elliptic machinery developed in [10] and [17]. The $C^{4 . \alpha}$ estimates follow from $C^{2, \alpha}$ estimates and the standard elliptic theory. Finally, we make use of the $C^{4, \alpha}$ estimates and the degree theory introduced in [18] to prove the existence of at least one solution of (6).

Proposition 1.1. Suppose that $f, \psi$ satisfy (1), (2), (5), (7), and (8). Then any solution $u$ of $(6)$ with $\lambda(\operatorname{Id}+H(u)) \in \Gamma, a_{1} \leqq u \leqq A_{1}$, satisfies either $a_{1}<u<A_{1}$ or $u \equiv a_{1}$ or $u \equiv A_{1}$.

Proposition 1.2. Suppose that $f, \psi$ satisfy (1), (2), (5), (7), and (8). Then there exists a positive constant $C_{1}$ depending only on $\delta_{0}, a_{1}, A_{1}, f, \psi$, such that

$$
|\nabla u|_{C^{0}} \leqq C_{1},
$$

for any solution $u$ of $(6)$ with $\lambda(\mathrm{Id}+H(u)) \in \Gamma, a_{1} \leqq u \leqq A_{1}$. 
Proof of Proposition 1.1: Let $\bar{u}=A_{1}$, we know from (7) that

$$
f(\lambda(\mathrm{Id}+H(\bar{u}))) \leqq \psi(x, \bar{u}) .
$$

Therefore, $\bar{u}-u \geqq 0$ satisfies $L(\bar{u}-u) \leqq 0$, where $L$ is a linear second-order elliptic operator. According to the maximum principle, either $\bar{u}-u>0$ or $\bar{u}-u \equiv 0$, i.e., $u<A_{1}$ or $u \equiv A_{1}$. Similarly we can prove that either $u>a_{1}$ or $u \equiv a_{1}$.

In order to prove Proposition 1.2 and to obtain the second derivative estimates of $u$, we need to make use of the following function $\varphi(s)$ defined on $a_{1} \leqq s \leqq A_{1}$ :

$$
\varphi(s)=\frac{1}{16 M_{0}^{2}}\left(M_{0}-s\right)^{2}, \quad a_{1} \leqq s \leqq A_{1},
$$

where $M_{0}=2\left(\left|a_{1}\right|+\left|A_{1}\right|\right)+1$.

By a simple calculation we obtain

$$
\begin{gathered}
\varphi^{\prime}(s)=\frac{s-M_{0}}{8 M_{0}^{2}} \in\left(-\frac{3}{16 M_{0}},-\frac{1}{16 M_{0}}\right), \\
\varphi^{\prime \prime}(s)=\frac{1}{8 M_{0}^{2}} \\
\varphi^{\prime \prime}(s)-2 \varphi^{\prime}(s)^{2} \geqq \frac{1}{32 M_{0}^{2}} .
\end{gathered}
$$

Proof of Proposition 1.2: Let

$$
h(x)=v e^{\varphi(u)},
$$$$
x \in M,
$$

where $v=\sqrt{1+|\nabla u|^{2}}$ and $\nabla u$ is the gradient of $u$.

Since $h$ is a smooth function defined on a compact manifold $M$, it attains its maximum at some point $x_{0} \in M$, i.e.,

$$
h\left(x_{0}\right)=\max _{x \in M} h(x) .
$$

The control of $|\nabla u|$ follows immediately from the control of $h\left(x_{0}\right)$, which is what we shall obtain in what follows.

Choose a normal coordinate system near $x_{0}$, namely, a coordinate system $\left\{x^{1}, \cdots, x^{n}\right\}$ with $g_{i j}\left(x_{0}\right)=\delta_{i j}, \Gamma_{i j}^{k}\left(x_{0}\right)=0$, where $g=g_{i j} d x^{i} d x^{j}$ is the Riemannian metric and $\Gamma_{i j}^{k}$ the Christoffel symbol; see [3]. Here and in what follows repeated indices denote sum over the indices. 
Without loss of generality we assume that $\left(u_{i j}\left(x_{0}\right)\right)$ is a diagonal matrix. It is well known that

$$
|\nabla u|^{2}=g^{s t} u_{s} u_{t}
$$

and

$$
H(u)_{i j}=\left(u_{l m}-u_{t} \Gamma_{l m}^{\prime}\right) B^{l i} B^{m j},
$$

where $u_{s}=\partial u / \partial x^{s},\left(g^{i j}\right)$ is the inverse matrix of $\left(g_{i j}\right),\left(B^{i j}\right)$ is the square root matrix of $\left(g^{i j}\right)$.

Because of (9), we have, at $x_{0}$,

$$
\begin{aligned}
& 0=\frac{h_{i}}{h}, \\
& 0 \geqq\left(\frac{h_{i}}{h}\right)_{i} .
\end{aligned}
$$

Clearly, $h_{i}, h_{i i}$ involve $u_{i}, u_{i i}$; therefore we calculate $v_{i}$ and $v_{i i}$ first:

$$
v^{2}=1+g^{s t} u_{s} u_{t}
$$

Applying $\partial / \partial x^{i}$ to the above, we have

$$
2 v v_{i}=\partial_{i} g^{s t} u_{s} u_{t}+2 g^{s t} u_{s i} u_{t}
$$

Since we are working with the normal coordinate system we have, at $x_{0}, \Gamma_{i j}^{k}=0$, $\partial_{k} g^{i j}=0, \partial_{k} B^{i j}=0, \partial_{k} g_{i j}=0$. We apply $\partial / \partial x^{i}$ to (12) and evaluate it at $x_{0}$, obtaining

$$
\begin{aligned}
2 v_{i}^{2}+2 v v_{i i} & =\partial_{i i} g^{s t} u_{s} u_{t}+2 g^{s t} u_{s i i} u_{t}+2 g^{s t} u_{s i} u_{t i} \\
& =\partial_{i i} g^{s t} u_{s} u_{t}+2 \sum_{s} u_{s i i} u_{s}+2 u_{i i}^{2} \\
v_{i i} & =\frac{1}{2 v}\left(-2 v_{i}^{2}+\partial_{i i} g^{s t} u_{s} u_{t}+2 \sum_{s} u_{s i i} u_{s}+2 u_{i i}^{2}\right)
\end{aligned}
$$

Evaluating (12) at $x_{0}$ we have

$$
v_{i}=\frac{1}{v} u_{i i} u_{i}
$$

In view of (10) and (11), the following equations hold at $x_{0}$ :

$$
\begin{gathered}
0=\frac{h_{i}}{h}=\frac{v_{i}}{v}+\varphi^{\prime}(u) u_{i}, \\
0 \geqq\left(\frac{h_{i}}{h}\right)_{i}=\frac{v_{i i}}{v}-\left(\frac{v_{i}}{v}\right)^{2}+\varphi^{\prime \prime}(u) u_{i}^{2}+\varphi^{\prime}(u) u_{i i} .
\end{gathered}
$$


Multiplying (16) by $f_{\lambda_{1}}$ and summing over $i$, we get

$$
0 \geqq \frac{1}{v} \sum_{i} f_{\lambda_{1}} v_{i i}-\sum_{i} f_{\lambda_{i}}\left(\frac{v_{i}}{v}\right)^{2}+\varphi^{\prime \prime}(u) \sum_{i} f_{\lambda_{t}} u_{i}^{2}+\varphi^{\prime}(u) \sum_{i} f_{\lambda_{i}} u_{i i} .
$$

It follows from (13) and (17) that

$$
\begin{aligned}
0 \geqq & \frac{1}{2 v^{2}}\left\{\sum_{i} f_{\lambda_{1}} \partial_{i i} g^{s t} u_{s} u_{t}+2 \sum_{s, i} f_{\lambda_{i}} u_{s i} u_{s}+2 \sum_{i} f_{\lambda_{i}} u_{i i}^{2}\right\} \\
& -2 \sum_{i}\left(\frac{v_{i}}{v}\right)^{2} f_{\lambda_{1}}+\varphi^{\prime \prime}(u) \sum_{i} f_{\lambda_{1}} u_{i}^{2}+\varphi^{\prime}(u) \sum_{i} f_{\lambda_{1}} u_{i i}, \\
0 \geqq & \frac{1}{2 v^{2}}\left\{\sum_{i} f_{\lambda_{1}} \partial_{i i} g^{s t} u_{s} u_{i}+2 \sum_{i, s} f_{\lambda_{1}} u_{s i i} u_{s}+2 \sum_{i} f_{\lambda_{i}} u_{i i}^{2}\right\} \\
& +\left(\varphi^{\prime \prime}(u)-2 \varphi^{\prime}(u)^{2}\right) \sum_{i} f_{\lambda_{1}} u_{i}^{2}+\varphi^{\prime}(u) \sum_{i} f_{\lambda_{1}} u_{i i} .
\end{aligned}
$$

We differentiate the equation

$$
f\left(\lambda\left(\delta_{i j}+\left(u_{l m}-u_{i} \Gamma_{l m}^{\prime}\right) B^{\prime i} B^{m \jmath}\right)\right)=\psi(x, u)
$$

in the $x^{s}$ direction and obtain

$$
\begin{aligned}
F_{u_{i},}\left\{\left(u_{l m}-u_{t} \Gamma_{l m}^{\prime}\right)_{s} B^{l i} B^{m j}\right. & +\left(u_{l m}-u_{t} \Gamma_{l m}^{\prime}\right) \partial_{s} B^{l i} B^{m j} \\
& \left.+\left(u_{l m}-u_{t} \Gamma_{l m}^{\prime}\right) B^{i i} \partial_{s} B^{m j}\right\} \\
=\psi_{s}+\psi_{u} u_{s} . &
\end{aligned}
$$

Differentiating (20) again and evaluating it at $x_{0}$ we see, by using the concavity of $F$, that

$$
\begin{gathered}
\sum_{i} F_{u_{i t}}\left\{\left(u_{i i}-u_{i} \Gamma_{i i}^{t}\right)_{s s}+u_{i i} \partial_{s s} B^{i i}+u_{i i} \partial_{s s} B^{i i}\right\} \\
\geqq \psi_{s s}+2 \psi_{s u} u_{s}+\psi_{u u} u_{s}^{2}+\psi_{u} u_{s s}, \\
\sum_{i} f_{\lambda_{1}}\left\{u_{i i s s}-2 u_{s s} \partial_{s} \Gamma_{i i}^{s}-u_{t} \partial_{s s} \Gamma_{i i}^{\prime}+2 u_{i i} \partial_{s s} B^{i i}\right\} \\
\geqq \psi_{s s}+2 \psi_{s u} u_{s}+\psi_{u u} u_{s}^{2}+\psi_{u} u_{s s} .
\end{gathered}
$$

We evaluate (20) at $x_{0}$ :

$$
\sum_{i} f_{\lambda_{i}}\left(u_{i i s}-u_{t} \partial_{s} \Gamma_{i i}^{t}\right)=\psi_{s}+\psi_{u} u_{s},
$$


and substitute (22) into (18):

$$
\begin{aligned}
C \geqq & \frac{1}{2 v^{2}}\left\{\sum_{i} f_{\lambda_{1}} \partial_{i i} g^{s t} u_{s} u_{t}+2 \sum_{i, s} f_{\lambda_{1}} u_{s} u_{t} \partial_{s} \Gamma_{i i}^{t}+2 \sum_{i} f_{\lambda_{1}} u_{i i}^{2}\right\} \\
& +\left(\varphi^{\prime \prime}(u)-2 \varphi^{\prime}(u)^{2}\right) \sum_{i} f_{\lambda_{i}} u_{i}^{2}+\varphi^{\prime}(u) \sum_{i} f_{\lambda_{i}} u_{i i}
\end{aligned}
$$

Here and in what follows, $C$ is some constant depending on $M_{0}, \delta_{0}, f, \psi$.

We deduce from (1) and (2) that

$$
\begin{aligned}
\sum_{i} f_{\lambda_{i}} u_{i i} & =-\sum_{i} f_{\lambda_{i}}+\sum_{i} f_{\lambda_{i}} \lambda_{i} \\
& \leqq-\sum_{i} f_{\lambda_{i}}+f(\lambda)-\lim _{\substack{\lambda \rightarrow 0 \\
\lambda \in \Gamma}} \inf f(\lambda) \\
& \leqq-\sum_{i} f_{\lambda_{i}}+C .
\end{aligned}
$$

Noting that

$$
-\frac{3}{16 M_{0}} \leqq \varphi^{\prime}(u) \leqq-\frac{1}{16 M_{0}}
$$

we obtain

$$
\begin{aligned}
\varphi^{\prime}(u) \sum_{i} f_{\lambda_{i}} u_{i i} & \geqq-\varphi^{\prime}(u) \sum_{i} f_{\lambda_{i}}+C \varphi^{\prime}(u) \\
& \geqq \frac{1}{16 M_{0}} \sum_{i} f_{\lambda_{i}}-C .
\end{aligned}
$$

We substitute (24) into (23) and use the fact that $\varphi^{\prime \prime}(u)-2 \varphi^{\prime}(u)^{2} \geqq 1 / 32 M_{0}^{2}$; then

$$
\begin{aligned}
C \geqq & \frac{1}{2 v^{2}} \sum_{i, s, t} f_{\lambda_{i}}\left(\partial_{i i} g^{s t}+2 \partial_{s} \Gamma_{i i}^{t}\right) u_{s} u_{t} \\
& +\frac{1}{32 M_{0}^{2}} \sum_{i} f_{\lambda_{i}} u_{i}^{2}+\frac{1}{16 M_{0}} \sum_{i} f_{\lambda_{i}}
\end{aligned}
$$

By a simple calculation we see that, at $x_{0}$,

$$
\begin{gathered}
\partial_{i i} g^{s t}=-\partial_{i} \Gamma_{i t}^{s}-\partial_{i} \Gamma_{i s}^{t}, \\
R_{s i t i}=R_{i t i}^{s}=\partial_{t} \Gamma_{i i}^{s}-\partial_{i} \Gamma_{i t}^{s},
\end{gathered}
$$

where

$$
\begin{gathered}
R_{k i j}^{\prime}=\partial_{i} \Gamma_{j k}^{l}-\partial_{j} \Gamma_{i k}^{\prime}+\Gamma_{i m}^{l} \Gamma_{j k}^{m}-\Gamma_{j m}^{\prime} \Gamma_{i k}^{m} \\
R_{l k i j}=g_{l m} R_{k i j}^{m} .
\end{gathered}
$$


If we use the hypothesis that $(M, g)$ has non-negative sectional curvature we can conclude that

$$
\begin{aligned}
\sum_{s, t}\left(\partial_{i i} g^{s t}+2 \partial_{s} \Gamma_{i i}^{t}\right) u_{s} u_{t} & =\sum_{s, t}\left(-\partial_{i} \Gamma_{i t}^{s}-\partial_{i} \Gamma_{i s}^{t}+2 \partial_{s} \Gamma_{i i}^{t}\right) u_{s} u_{t} \\
& =2 \sum_{s, t}\left(-\partial_{i} \Gamma_{i t}^{s}+\partial_{s} \Gamma_{i i}^{t}\right) u_{s} u_{t} \\
& =2 \sum_{s, t}\left(-\partial_{i} \Gamma_{i t}^{s}+\partial_{t} \Gamma_{i i}^{s}\right) u_{s} u_{t} \\
& =2 \sum_{s, t} R_{s i t i} u_{s} u_{t} \\
& \geqq 0 .
\end{aligned}
$$

Therefore we deduce from (23) that

$$
C \geqq \frac{1}{32 M_{0}^{2}} \sum_{i} f_{\lambda_{i}} u_{i}^{2}+\frac{1}{16 M_{0}} \sum_{i} f_{\lambda_{i}} .
$$

Because of (5),

$$
|\lambda| \leqq C .
$$

Therefore,

$$
f_{\lambda_{i}} \geqq \frac{1}{C}, \quad i=1, \cdots, n
$$

It follows from (26) that

$$
\begin{gathered}
|\nabla u|^{2}=\sum_{i} u_{i}^{2} \leqq C, \\
h \leqq h\left(x_{0}\right) \leqq C .
\end{gathered}
$$

We have completed the proof of Proposition 1.2.

In the following we estimate second derivatives of the solution $u$ of (6). The bound will depend on the maximum norm of $u$ and $\nabla u$.

Proposition 1.3. Suppose that $f, \psi$ satisfy (1), (2), (5), (7) and (8), $u$ is any solution of (6) with $\lambda(\mathrm{Id}+H(u)) \in \Gamma, a_{1} \leqq u \leqq A_{1},|\nabla u| \leqq C_{1}$. Then there exists a positive constant $C_{2}$ depending only on $\delta_{0}, a_{1}, A_{1}, C_{1}, f, \psi$, such that

$$
\left|\nabla^{2} u\right|_{C^{0}} \leqq C_{2}
$$


Proof of Proposition 1.3: Let $\left(H(u)_{i j}(x)\right)$ denote the Hessian matrix under some orthonormal basis at $x$ and

$$
\Lambda:=\max _{x \in M} \max _{\substack{|\alpha|=1 \\ \alpha \in R^{n}}}\left(\delta_{i j}+H(u)_{i j}(x)\right) \alpha^{i} \alpha^{j} \exp \left\{\frac{1}{2} a|\nabla u|^{2}\right\} e^{\Phi(u)},
$$

where $\varphi$ is the function defined in Section 2 and $a$ is some positive constant to be determined later; $a$ will be small and will depend on the maximum norm of $u$, $\nabla u$.

Clearly, $\Lambda$ is achieved at some point $x_{0} \in M$ along some direction $\alpha,|\alpha|=1$.

We choose a normal coordinate at $x_{0}$ and without loss of generality we can assume that $\left(u_{i j}\left(x_{0}\right)\right)$ is a diagonal matrix and $\alpha=(1,0, \cdots, 0)$. Therefore,

$$
\Lambda=\left(1+u_{11}\left(x_{0}\right)\right) \exp \left\{\frac{1}{2} a|\nabla u|^{2}\right\} e^{\varphi(u)} .
$$

Let

$$
w:=\left(1+\left(u_{l m}-u_{t} \Gamma_{l m}^{\prime}\right) B^{\prime 1} B^{m l}\right) \exp \left\{\frac{1}{2} a|\nabla u|^{2}\right\} e^{\Phi(u)} .
$$

Clearly, $w$ has a local maximum at $x_{0}$. Hence

$$
\begin{aligned}
& 0=\frac{w_{i}}{w}, \\
& 0 \geqq\left(\frac{w_{i}}{w}\right)_{i}
\end{aligned}
$$

A calculation yields that, at $x_{0}$,

$$
\begin{aligned}
0 & =\frac{w_{i}}{w} \\
& =\frac{\left\{\left(u_{l m}-u_{t} \Gamma_{l m}^{l}\right) B^{\prime 1} B^{m 1}\right\}_{i}}{1+\left(u_{l m}-u_{t} \Gamma_{l m}^{\prime}\right) B^{\prime 1} B^{m l}}+\frac{1}{2} a \partial_{i} g^{s t} u_{s} u_{t}+a g^{s t} u_{s i} u_{t}+\varphi^{\prime}(u) u_{i},
\end{aligned}
$$

i.e.,

$$
\begin{gathered}
0=\frac{u_{11 i}-u_{t} \partial_{i} \Gamma_{11}^{t}}{1+u_{11}}+a u_{i i} u_{i}+\varphi^{\prime}(u) u_{i}, \\
0 \geqq\left(\frac{w_{i}}{w}\right)_{i} \\
\geqq \frac{\left\{\left(u_{l m}-u_{t} \Gamma_{l m}^{t}\right) B^{\prime 1} B^{m 1}\right\}_{i i}}{1+u_{11}}-\left(\frac{\left\{\left(u_{l m}-u_{t} \Gamma_{l m}^{\prime}\right) B^{\prime 1} B^{m l}\right\}_{i}}{1+u_{11}}\right)^{2} \\
+\frac{1}{2} a \partial_{i i} g^{s t} u_{s} u_{t}+a \sum_{s} u_{s i i} u_{s}+a u_{i i}^{2}+\varphi^{\prime \prime}(u) u_{i}^{2}+\varphi^{\prime}(u) u_{i i} \\
=\frac{u_{11 i i}-2 u_{i i} \partial_{i} \Gamma_{11}^{i}-u_{t} \partial_{i i} \Gamma_{11}^{t}+2 u_{11} B_{i i}^{11}}{\lambda_{1}}-\left(a u_{i i} u_{i}+\varphi^{\prime}(u) u_{i}\right)^{2} \\
+\frac{1}{2} a \partial_{i i} g^{s t} u_{s} u_{t}+a \sum_{s} u_{s i i} u_{s}+a u_{i i}^{2}+\varphi^{\prime \prime}(u) u_{i}^{2}+\varphi^{\prime}(u) u_{i i} .
\end{gathered}
$$


Multiplying the above inequality by $f_{\lambda_{i}}$ and summing over $i$, one obtains

$$
\begin{aligned}
C+C & \frac{\sum_{i} f_{\lambda_{1}}}{\lambda_{1}} \\
\geqq & \frac{\sum_{i}\left(f_{\lambda_{1}} u_{11 i i}-2 f_{\lambda_{i}} u_{i i} \partial_{i} \Gamma_{11}^{i}+2 f_{\lambda_{1}} u_{11} \partial_{i i} B^{11}\right)}{\lambda_{1}}-a^{2} \sum_{i} f_{\lambda_{i}} u_{i i}^{2} u_{i}^{2} \\
& -2 a \varphi^{\prime}(u) \sum_{i} f_{\lambda_{1}} u_{i i} u_{i}^{2}-\varphi^{\prime}(u)^{2} \sum_{i} f_{\lambda_{1}} u_{i}^{2}+\frac{1}{2} a \sum_{i} f_{\lambda_{t}} \partial_{i i} g^{s t} u_{s} u_{i} \\
& +a \sum_{i, s} f_{\lambda_{1}} u_{s i i} u_{s}+a \sum_{i} f_{\lambda_{1}} u_{i i}^{2}+\varphi^{\prime \prime}(u) \sum_{i} f_{\lambda_{1}} u_{i}^{2}+\varphi^{\prime}(u) \sum_{i} f_{\lambda_{1}} u_{i i} .
\end{aligned}
$$

Using (21), (22), (24) and the above inequality, we have

$$
\begin{aligned}
& C+C \frac{\sum_{i} f_{\lambda_{i}}}{\lambda_{i}} \\
& \geqq \frac{1}{\lambda_{1}} \sum_{i}\left(2 f_{\lambda_{1}} u_{11} \partial_{1} \Gamma_{i i}^{1}-2 f_{\lambda_{1}} u_{i i} \partial_{11} B^{i i}-2 f_{\lambda_{1}} u_{i i} \partial_{i} \Gamma_{11}^{i}+2 f_{\lambda_{t}} u_{11} \partial_{i i} B^{11}\right) \\
& -a^{2} \sum_{i} f_{\lambda_{i}} u_{i i}^{2} u_{i}^{2}-2 a \varphi^{\prime}(u) f_{\lambda_{i}} u_{i} u_{i}^{2}+\left(\varphi^{\prime \prime}(u)-\varphi^{\prime}(u)^{2}\right) \sum_{i} f_{\lambda_{i}} u_{i}^{2} \\
& +\frac{1}{2} a \sum_{i} f_{\lambda_{1}} \partial_{i i} g^{s t} u_{s} u_{t}+a \sum_{i} f_{\lambda_{i}} u_{s} u_{\imath} \partial_{s} \Gamma_{i i}^{t}+a \sum_{i} f_{\lambda_{i}} u_{i i}^{2}+\frac{1}{16 M_{0}} \sum_{i} f_{\lambda_{i}} \\
& C+C \frac{\sum_{i} f_{\lambda_{1}}}{\lambda_{1}} \geqq 2 \sum_{i} f_{\lambda_{1}}\left(\partial_{1} \Gamma_{i i}^{1}+\partial_{i i} B^{11}\right)-\frac{C}{\lambda_{1}} \sum_{i} f_{\lambda_{1}}\left|u_{i i}\right| \\
& -a^{2} \sum_{i} f_{\lambda_{i}} u_{i i}^{2} u_{i}^{2}-2 a \varphi^{\prime}(u) \sum_{i} f_{\lambda_{1}} u_{i i} u_{i}^{2} \\
& +\frac{1}{2} a \sum_{i} f_{\lambda_{s}} u_{s} u_{s}\left(\partial_{i i} g^{s t}+2 \partial_{s} \Gamma_{i i}^{i}\right)+a \sum_{i} f_{\lambda_{i}} u_{i i}^{2}+\frac{1}{16 M_{0}} \sum_{i} f_{\lambda_{i}}
\end{aligned}
$$

By a simple calculation, we get

$$
\partial_{i i} B^{11}=\frac{1}{2} \partial_{i i} g^{11}
$$


As before, using the fact that $(M, g)$ has non-negative sectional curvature, we have

$$
\begin{gathered}
\partial_{1} \Gamma_{i i}^{1}+\partial_{i i} B^{11}=\partial_{1} \Gamma_{i i}^{1}+\frac{1}{2} \partial_{i i} g^{11} \geqq 0, \\
\left(\partial_{i i} g^{s t}+2 \partial_{s} \Gamma_{i i}^{t}\right) u_{s} u_{t} \geqq 0 .
\end{gathered}
$$

Thus,

$$
\begin{aligned}
C+C \frac{\sum_{i} f_{\lambda_{1}}}{\lambda_{1}} \geqq & -\frac{C}{\lambda_{1}} \sum_{i} f_{\lambda_{i}}\left|u_{i i}\right|-a^{2} \sum_{i} f_{\lambda_{1}} u_{i i}^{2} u_{i}^{2} \\
& -2 a \varphi^{\prime}(u) \sum_{i} f_{\lambda_{i}} u_{i i} u_{i}^{2}+a \sum_{i} f_{\lambda_{i}} u_{i i}^{2}+\frac{1}{16 M_{0}} \sum_{i} f_{\lambda_{i}} .
\end{aligned}
$$

Since

$$
\left|a^{2} \sum_{i} f_{\lambda_{i}} u_{i i}^{2} u_{i}^{2}\right| \leqq a^{2} C \sum_{i} f_{\lambda_{i}} u_{i i}^{2}
$$

we can take $a$ so small that

$$
a C<\frac{1}{2}
$$

and then

$$
a \sum_{i} f_{\lambda,} u_{i i}^{2}-a^{2} \sum_{i} f_{\lambda} u_{i i}^{2} u_{i}^{2} \geqq \frac{1}{2} a \sum_{i} f_{\lambda} u_{i i}^{2}
$$

Therefore,

$$
\begin{aligned}
& -\frac{C}{\lambda_{1}} \sum_{i} f_{\lambda_{1}}\left|u_{i i}\right|-a^{2} \sum_{i} f_{\lambda_{i}} u_{i i}^{2} u_{1}^{2}+a \sum_{i} f_{\lambda_{1}} u_{i i}^{2} \\
& \geqq \frac{1}{2} a \sum_{i} f_{\lambda_{i}} u_{i i}^{2}-\frac{C}{\lambda_{1}} \sum_{i} f_{\lambda_{i}}\left|u_{i j}\right| \\
& \quad=\sum_{i} f_{\lambda_{i}}\left|u_{i i}\right|\left(\frac{1}{4} a\left|u_{i i}\right|-\frac{C}{\lambda_{1}}\right)+\frac{1}{4} a \sum_{i} f_{\lambda_{1}} u_{i i}^{2} \\
& \geqq-C \sum_{a / 4\left|u_{i}\right| \leqq C / \lambda_{1}} f_{\lambda_{i}} \frac{\left|u_{i i}\right|}{\lambda_{1}}+\frac{1}{4} a \sum_{i} f_{\lambda_{1}} u_{i i}^{2} \\
& \geqq-\frac{C}{a \lambda_{1}^{2}} \sum_{i} f_{\lambda_{i}}+\frac{1}{4} a \sum_{i} f_{\lambda_{i}} u_{i i}^{2} .
\end{aligned}
$$


244

YANYAN LI

It follows from (31) and the above estimate that

$$
\begin{aligned}
C+ & \frac{C}{\lambda_{1}} \sum_{i} f_{\lambda_{1}}+\frac{C}{a \lambda_{1}^{2}} \sum_{i} f_{\lambda_{1}} \\
& \geqq-2 a \varphi^{\prime}(u) \sum_{i} f_{\lambda_{1}} u_{i i} u_{i}^{2}+\frac{1}{4} a \sum_{i} f_{\lambda_{1}} u_{i i}^{2}+\frac{1}{16 M_{0}} \sum_{i} f_{\lambda_{i}} .
\end{aligned}
$$

Clearly,

$$
\begin{aligned}
\left|2 a \varphi^{\prime}(u) \sum_{i} f_{\lambda_{i}} u_{i i} u_{i}^{2}\right| & \leqq C a \sum_{i} f_{\lambda_{i}}\left|u_{i i}\right| \\
& \leqq C a \sum_{i} f_{\lambda_{i}}+\frac{1}{4} a \sum_{i} f_{\lambda_{1}}\left|u_{i i}\right|^{2}
\end{aligned}
$$

Thus

$$
C+\frac{C}{\lambda_{1}} \sum_{i} f_{\lambda_{i}}+\frac{C}{a \lambda_{1}^{2}} \sum_{i} f_{\lambda_{i}} \geqq-C a \sum_{i} f_{\lambda_{i}}+\frac{1}{16 M_{0}} \sum_{i} f_{\lambda_{i}}
$$

We take $a$ so small that

$$
\frac{1}{32 M_{0}} \geqq C a
$$

Then,

$$
\frac{1}{32 M_{0}} \sum_{i} f_{\lambda_{i}} \leqq C+\frac{C}{\lambda_{1}} \sum_{i} f_{\lambda_{i}}+\frac{C}{a \lambda_{1}^{2}} \sum_{i} f_{\lambda_{i}}
$$

Now we fix $a$ which satisfies (32) and (34). Clearly, $a$ is under control. If

$$
\frac{C}{\lambda_{1}}+\frac{C}{a \lambda_{1}^{2}} \geqq \frac{1}{64 M_{0}}
$$

we have

$$
\lambda_{1} \leqq C .
$$

Since $\lambda_{1}+\cdots+\lambda_{n} \geqq-n$ and $\lambda_{1}$ is the largest among $\left\{\lambda_{1}, \cdots, \lambda_{n}\right\}$, (36) gives the bound of $|\lambda|$. Otherwise we have

$$
\frac{C}{\lambda_{1}}+\frac{C}{a \lambda_{1}^{2}} \leqq \frac{1}{64 M_{0}} .
$$


It then follows from (35) and (37) that

$$
\frac{1}{64 M_{0}} \sum_{i} f_{\lambda_{i}} \leqq C,
$$

which, according to (5), gives a bound for $|\lambda|$.

In any case, we have obtained

$$
|\lambda| \leqq C
$$

which gives an a priori bound for second derivatives of $u$.

Proof of Theorem 1.1: Without loss of generality we assume that $a_{1}<0<A_{1}$ and $u \equiv a_{1}$ or $u \equiv A_{1}$ is not a solution of (6). Choose $\varepsilon>0$ small enough, such that

$$
f(1, \cdots, 1)+\varepsilon a_{1}>\delta_{0}
$$

The above is possible since $f(1, \cdots, 1)>\delta_{0}$; see (7) and (8). Consider the following equation for $0 \leqq t \leqq 1$ :

$$
f(\lambda(\mathrm{Id}+H(u)))=t \psi(x, u)+(1-t)(\varepsilon u+f(1, \cdots, 1)), \quad x \in M .
$$

Since $u \equiv a_{1}$ and $u \equiv A_{1}$ are not solutions of (6), they are not solutions of (39). Therefore, according to Proposition 1.1, any solution $u^{\prime}$ of (39) with $a_{1} \leqq A_{1}$, $\lambda(\mathrm{Id}+H(u)) \in \Gamma$ satisfies $a_{1}<u<A_{1}$. It follows from the a priori estimates we have obtained that there exists some constant $R$, such that

$$
\left\|u^{\prime}\right\|_{c^{4 . a}} \leqq R-1<+\infty \text {. }
$$

Notice that $R$ is independent of $t$.

Let

$$
\begin{gathered}
O^{*}:=\left\{a_{1}<u<A_{1}\right\} \cap\left\{\|u\|_{c^{4 . a}}<R\right\} \\
\cap\left\{\lambda(\operatorname{Id}+H(u)) \in \Gamma, f\left(\lambda(\mathrm{Id}+H(u))>\delta_{0}\right\},\right. \\
H^{*}(t, u):=f(\lambda(\operatorname{Id}+H(u))-t \psi(x, u)-(1-t)(\varepsilon u+f(1, \cdots, 1)),
\end{gathered}
$$

where $0 \leqq t \leqq 1$. It is clear that

$$
H^{*}(t, u) \neq 0 \text { for all } 1 \leqq t \leqq 1, u \in \partial O^{*} .
$$

Therefore we can apply the degree theory introduced in [18]; according to the 
homotopy invariance of the degree, we have

$$
\operatorname{deg}\left(H^{*}(0, \cdot), O^{*}, 0\right)=\operatorname{deg}\left(H^{*}(1, \cdot), O^{*}, 0\right)
$$

Since

$$
H^{*}(0, u)=f(\lambda(\mathrm{Id}+H(u))-\varepsilon u-f(1, \cdots, 1),
$$

it is obvious that $u \equiv 0$ is the only solution of $H^{*}(0, u)=0$ and the linearized operator at $u \equiv 0$ is invertible. Therefore,

$$
\begin{aligned}
& \operatorname{deg}\left(H^{*}(0, \cdot), O^{*}, 0\right)= \pm 1 \\
& \operatorname{deg}\left(H^{*}(1, \cdot), O^{*}, 0\right)= \pm 1
\end{aligned}
$$

Hence (6) has a solution in $O^{*}$.

\section{Further Results on an $n$-Dimensional Flat Torus $T^{n}$}

In this section we study the existence and uniqueness of solutions of certain nonlinear elliptic equations on $T^{n}$, the standard $n$-dimensional flat torus.

Consider the following equation:

$$
f\left(\lambda\left(\tilde{a}_{i j}(x)+u_{i j}(x)\right)\right)=\psi(x, u(x)), \quad x \in T^{n},
$$

where a function on $T^{n}$ is viewed as a function on $\mathbf{R}^{n}$ which is one-periodic in each variable, $\left(u_{i j}\right)$ denotes the Hessian matrix of $u$, and $\left(\tilde{a}_{i j}(x)\right)>0$ is a given $n \times n$ smooth positive definite matrix function; $\lambda\left(\tilde{a}_{i j}+u_{i j}\right)$ denotes the eigenvalues of the matrix $\left(\tilde{a}_{i j}+u_{i j}\right), \lambda=\left(\lambda_{1}, \cdots, \lambda_{n}\right)$.

$\psi$ is a smooth function satisfying the following conditions: There exists two constants $m<M$, such that

$$
\psi(x, m) \leqq f\left(\lambda\left(\tilde{a}_{i j}(x)\right)\right) \leqq \psi(x, M) \text { for all } x \in T^{n}
$$

There exists a $\delta_{0}>0$, such that

$$
\psi(x, s)>\delta_{0}>\lim _{\lambda \rightarrow \lambda_{0}} \sup f(\lambda) \text { for all } \lambda_{0} \in \partial \Gamma, x \in T^{n}, m \leqq s \leqq M
$$

THEOREM 2.1. Suppose that $f$ and $\psi$ satisfies (1), (2), (5), (42) and (43), where $\left(\tilde{a}_{i j}(x)\right)>0$ is a smooth $n \times n$ positive definite matrix function defined on $T^{n}$. Then (41) has a smooth solution $u$ with $m \leqq u \leqq M, \lambda\left(\tilde{a}_{i j}(x)+u_{i j}(x)\right) \in \Gamma$ for all $x \in T^{n}$. If $\psi_{u} \geqq 0$, such a solution is unique up to constants.

The proof of the above result is almost the same as the proof of Theorem 1.1. 
In the following we study a simpler equation on $T^{n}$, and in this case we have necessary and sufficient conditions for the existence and uniqueness of solutions. Consider

$$
\operatorname{det}\left(a_{i j}+u_{i j}(x)\right)=\psi(x), \quad x \in T^{n},
$$

where $\left(a_{i j}\right)$ is a positive definite constant matrix, and $\psi(x)>0$ is a smooth positive function.

We have the following necessary condition for the existence of a solution to (44).

LEMMA 2.1. If (44) has a solution $u \in C^{2}\left(T^{n}\right)$, then

$$
\int_{T^{n}} \psi(x)=\int_{T^{n}} \operatorname{det}\left(a_{i j}\right)
$$

Proof: $\quad u$ can be viewed as a periodic function defined on $\mathbb{R}^{n}$ with period 1 in each variable. Let

$$
\begin{gathered}
v(x):=\frac{1}{2} \sum_{i, j} a_{i j} x^{i} x^{j}, \\
w=v+u,
\end{gathered}
$$

be functions defined on $\mathbf{R}^{n}$.

We have

$$
\begin{aligned}
\psi(x) d x^{1} \wedge \cdots \wedge d x^{n} & =\operatorname{det}\left(a_{i j}+u_{i j}(x)\right) d x^{1} \wedge \cdots \wedge d x^{n} \\
& =\operatorname{det}\left(w_{i j}(x)\right) d x^{1} \wedge \cdots \wedge d x^{n} \\
& =d w_{1} \wedge \cdots \wedge d w_{n} \\
& =d\left(v_{1}+u_{1}\right) \wedge \cdots \wedge d\left(v_{n}+u_{n}\right) \\
& =d v_{1} \wedge \cdots \wedge d v_{n}+\sum_{i=1}^{n} d u_{i} \wedge F_{i}
\end{aligned}
$$

where $v_{i}, u_{i}$ denote $\partial v / \partial x^{i}, \partial u / \partial x^{i}$, respectively, and

$$
\begin{aligned}
F_{i}= & (-1)^{i-1} d\left(v_{1}\right) \wedge \cdots \wedge d\left(v_{i-1}\right) \\
& \wedge d\left(v_{i+1}+u_{i+1}\right) \wedge \cdots \wedge d\left(v_{n}+u_{n}\right), \quad i=1,2, \cdots, n .
\end{aligned}
$$

Notice that $d v_{i}$ is well defined on $T^{n}$ even though $u_{i}$ is not. In fact,

$$
d v_{i}=\sum_{j} a_{i j} d x^{j}
$$


Therefore, $F_{i}$ is well defined on $T^{n}$ and $d F_{i}=0$; hence we have

$$
\begin{aligned}
\int_{T^{n}} d u_{i} \wedge F_{i} & =\int_{T^{n}} d\left(u_{i} \wedge F_{i}\right)=0, \\
\int_{T^{n}} \psi(x) & =\int_{\substack{0 \leqq x_{i} \leqq 1 \\
x \in \mathbf{R}^{n}}} d v_{1} \wedge \cdots \wedge d v_{n} \\
& =\int_{\substack{0 \leqq x_{i} \leqq 1 \\
x \in \mathbf{R}^{n}}}\left(\sum_{j_{1}} a_{i j_{i}} d x^{j_{1}}\right) \wedge \cdots \wedge\left(\sum_{j_{n}} a_{n j_{n}} d x^{j_{n}}\right) \\
& =\int_{\substack{0 \leqq x_{i} \leq 1 \\
x \in \mathbf{R}^{n}}} \operatorname{det}\left(a_{i j}\right) \\
& =\int_{T^{n}} \operatorname{det}\left(a_{i j}\right) .
\end{aligned}
$$

Equation (45) turns out also to be sufficient; namely, we have the following result:

THEOREM 2.2. Suppose that $\left(a_{i j}\right)>0$ is a positive definite constant matrix, $\psi(x)>0$ is a smooth positive function defined on $T^{n}$ satisfying (45). Then (44) has a smooth solution $u$ with $\left(a_{i j}+u_{i j}(x)\right)>0$ for all $x \in T^{n}$. Furthermore, such a solution is unique up to constants.

The uniqueness follows immediately from the maximum principle. To prove Theorem 2.2 we only need to prove the existence of a $C^{2, \mu}$ solution, $0<\mu<1$, since the smoothness of the solution follows from standard elliptic regularity theories.

Let

$$
\begin{gathered}
X^{2, \mu}:=\left\{u \in C^{2, \mu}\left(T^{n}\right) \mid \int_{T^{n}} u=0\right\}, \\
X^{\mu}:=\left\{u \in C^{\mu}\left(T^{n}\right) \mid \int_{T^{n}} u=0\right\},
\end{gathered}
$$

where $0<\mu<1$.

For $0 \leqq t \leqq 1$, consider

$$
\begin{aligned}
S_{i}(u) & :=\operatorname{det}\left(a_{i j}+u_{i j}(x)\right)-t \psi(x)-(1-t) \operatorname{det}\left(a_{i j}\right)=0 \text { on } T^{n} \\
u & \in X^{2, \mu}
\end{aligned}
$$


As we have proved before,

$$
S_{t}: X^{2, \mu} \rightarrow X^{\mu}
$$

Clearly, for any $u \in X^{2, \mu}$,

$$
S_{t}^{\prime}(u): X^{2, \mu} \rightarrow X^{\mu}
$$

By elliptic theory, $S_{t}^{\prime}(u)$ is an isomorphism from $X^{2, \mu}$ to $X^{\mu}$ for any $u \in X^{2, \mu}$ with $\left(a_{i j}+u_{i j}(x)\right)>0$ for all $x \in T^{n}$.

At $t=0, u \equiv 0$ is a solution of (46). Since the linearized operator $S_{t}^{\prime}(u)$ is an isomorphism from $X^{2 \mu}$ to $X^{\mu}$, in order to carry out the method of continuity we only need to obtain a priori estimates:

$$
|u|_{C^{2, \mu}\left(T^{n}\right)} \leqq C
$$

As we explained before we just have to ascertain that

$$
|u|_{C^{2}\left(T^{*}\right)} \leqq C
$$

We shall restrict ourselves to obtaining estimates for $t=1$ in (46), the same argument works for all $t$. We first estimate $|u|_{C^{0}}$. To do this we need the following elementary lemma.

Lemma 2.2. Let $w$ be a $C^{2}$ periodic function defined on $\mathbf{R}$ with period 1 , namely,

$$
w(r+1)=w(r) \text { for all } r \in \mathbb{R}
$$

We also assume that

$$
v(r):=w(r)+\frac{1}{2} M_{1} r^{2}
$$

is a convex function, i.e.,

$$
v^{\prime \prime}(r) \geqq 0 \quad \text { for all } r \in \mathbf{R} \text {, }
$$

where $M_{1}>0$ is some positive constant. Then

$$
\left|w\left(r_{1}\right)-w\left(r_{2}\right)\right| \leqq 6 M_{1} \text { for all } r_{1}, r_{2} \in \mathbb{R}
$$


Proof: Since $w$ is periodic, there exists a $\zeta \in(0,1]$, such that $w^{\prime}(\zeta)=0$. Therefore, for any $0<r_{1}<r_{2} \leqq 2$,

$$
\begin{aligned}
w\left(r_{2}\right)-w\left(r_{1}\right) & =\int_{r_{1}}^{r_{2}} w^{\prime}(t) d t \\
& =\int_{r_{1}}^{r_{2}} \int_{\zeta-1}^{t} w^{\prime \prime}(s) d s d t \\
& \geqq-M_{1} \int_{r_{1}}^{r_{2}} \int_{\zeta}^{t} d s d t \\
& \geqq-6 M_{1},
\end{aligned}
$$

which implies (47).

Since $\left(a_{1,}+u_{i}(x)\right)>0$, let $M_{1}=\max _{1 \leqq i, \jmath \leqq n}\left|a_{, j}\right|$; we know that, for all $1 \leqq i \leqq n$,

$$
\partial_{i t}\left(u+\frac{1}{2} M_{1}|x|^{2}\right) \geqq 0
$$

Therefore, by using Lemma $2.2 n$ times we have

$$
|u(x)-u(y)| \leqq 6 n M_{1} \text { for all } x, y \in T^{n}
$$

Since $\int_{r^{\prime \prime}} u(x)=0, u$ has to vanish somewhere; therefore,

$$
|u(x)| \leqq 12 n M_{1} . \quad x \in T^{n} .
$$

The gradient estimate is just a consequence of (48). The second derivative estimate follows from the proof of Theorem 1.1. This completes the proof.

\section{A Generalization of a Result in [5]}

Consider the following equation:

$$
\begin{gathered}
f\left(\lambda\left(u_{i j}(x)+\tilde{b}_{i j}(x)\right)\right)=\psi(x), \\
\left.u\right|_{\partial \Omega}=\varphi
\end{gathered}
$$

where $\Omega \subset \mathbb{R}^{n}$ is a smooth bounded domain, $\psi, \varphi,\left(\tilde{b}_{i j}\right)_{n \times n}$ are sufficiently smooth functions, and $f$ satisfies (1) and (2). We also assume that

$$
\psi>0 \text { in } \bar{\Omega} \text {. }
$$

Set

$$
0<\psi_{0}=\min _{\bar{\Omega}} \psi \leqq \max _{\bar{\Omega}} \psi=\psi_{1}
$$


We assume that, for some $\bar{\psi}_{0}<\psi_{0}$,

$$
\lim _{\lambda \rightarrow \lambda_{0}} \sup f(\lambda) \leqq \bar{\psi}_{0} \text { for all } \lambda_{0} \in \partial \Gamma
$$

In addition we assume that, for every $C>0$ and every compact set $K$ in $\Gamma$, there is a number $R=R(C, K)$, such that

$$
\begin{gathered}
f\left(\lambda_{1}, \cdots, \lambda_{n-1}, \lambda_{n}+R\right) \geqq C \text { for all } \lambda \in K, \\
f(R \lambda) \geqq C \text { for all } \lambda \in K .
\end{gathered}
$$

Using (2) and (51) we may conclude that there is a positive number $\delta$, such that

$$
\sum \lambda_{i} \geqq \delta \quad \text { in the set } \quad T=\left\{\lambda \in \Gamma: f(\lambda) \geqq \psi_{0}\right\}
$$

As explained in [7] we need some hypotheses on the shape of $\Omega$. Suppose that there exists a number $R$ sufficiently large such that, at every point $x \in \partial \Omega$, if $\kappa_{1}, \cdots, \kappa_{n-1}$ represent the principal curvatures of $\partial \Omega$ (relative to the interior normal), then

$$
\left(\kappa_{1}, \cdots, \kappa_{n-1}, R\right) \in \Gamma \text {. }
$$

Definition 3.1. $\Gamma$ is said to be of type 1 if the positive axes belong to $\partial \Gamma$, otherwise it is called of type 2.

Definition 3.2. A function $u \in C^{2}(\bar{\Omega})$ is called admissible if, at every $x \in \bar{\Omega}, \lambda\left(\tilde{b}_{i j}(x)+u_{i j}(x)\right) \in \Gamma$.

Our main result in this section is

THEOREM 3.1. Suppose that $f, \Omega, \psi$ satisfy (1), (2), (5), (50)-(53), and (54). Then (49) has a unique smooth admissible solution.

Remark 3.1. Theorem 3.1 is a generalization of results in [5] and [7]. The result is proved in [7] if $\tilde{b}_{i j} \equiv 0$ and in [5] if $f(\lambda)=\lambda_{1} \cdots \lambda_{n}$.

Proposition 3.1. (Existence of admissible solutions). There exists $a \underline{u}$ which is admissible and satisfies

$$
\begin{aligned}
\left.\underline{u}\right|_{\partial \Omega} & =\varphi \\
\underline{\psi}:=f\left(\lambda\left(\underline{u}_{i j}(x)+\tilde{b}_{i j}(x)\right)\right) & \geqq \psi_{1}+1 \quad \text { for all } x \in \bar{\Omega} .
\end{aligned}
$$


Proof: It has been proved in [7] (see Section 2 of [7]) that there exists some function $u \in C^{2}(\bar{\Omega})$, such that

$$
\begin{array}{cc}
\lambda\left(u_{i j}(x)\right) \in \Gamma, & x \in \bar{\Omega}, \\
\left.u\right|_{\partial \Omega}=0 &
\end{array}
$$

Let $\underline{u}=A u+\varphi$, where $\varphi$ has been extended smoothly to $\bar{\Omega}$. Since $\left\{\lambda\left(u_{i j}(x)\right): x \in \bar{\Omega}\right\}$ is a compact set in $\Gamma$, there exists a compact set $K \subset \Gamma$, such that, for any sufficiently large $A$,

$$
\left\{\lambda\left(u_{i j}(x)+A^{-1} \varphi_{i j}(x)+A^{-1} \tilde{b}_{i j}(x): x \in \bar{\Omega}\right\} \subset K .\right.
$$

Therefore, according to (53) we can choose $A$ so large that

$$
\begin{aligned}
\Psi & =f\left(\lambda\left(\underline{u}_{i j}(x)+\tilde{b}_{i j}(x)\right)\right) \\
& =f\left(A\left\{\lambda\left(u_{i j}(x)+A^{-1} \varphi_{i j}(x)+A^{-1} \tilde{b}_{i j}(x)\right)\right\}\right) \\
& \geqq \psi_{1}+1 .
\end{aligned}
$$

The uniqueness part of Theorem 3.1 follows easily from the following propositions, which have been proved in [7] and which for completeness we state and prove.

Proposition 3.2. Assume that $f$ satisfies (1) and $(2),\left(\tilde{b}_{i j}(x)\right)$ is some $n \times n$ continuous symmetric matrix function defined on $\bar{\Omega}$, where $\Omega$ is a bounded domain in $\mathbb{R}^{\prime \prime}$. Let $u$ be a $C^{2}$ function defined on $\Omega$ with $\lambda\left(\tilde{b}_{i j}(x)+u_{i j}(x)\right) \in \Gamma$ for all $x \in \Gamma, v$ be another $C^{2}$ function on $\Omega, \lambda\left(\tilde{b}_{i j}(x)+v_{i j}(x)\right)$ lying outside $\tilde{\Gamma}(x)$, where

$$
\tilde{\Gamma}(x):=\left\{\lambda \in \Gamma \mid f(\lambda) \geqq f\left(\lambda\left(\tilde{b}_{i j}(x)+u_{i j}(x)\right)\right)\right\}
$$

If $u \leqq v$ on $\partial \Omega$, then

$$
u \leqq v \quad \text { in } \Omega
$$

Proof: If this were not the case, $v-u$ would achieve a negative minimum at some point $x \in \Omega$. Since, as matrices, $\left\{v_{i j}\right\} \geqq\left\{u_{i j}\right\}$ at $x$, the eigenvalues of $\left\{\tilde{b}_{i j}(x)+v_{i j}(x)\right\}$ are not smaller than the corresponding ones for $\left\{\tilde{b}_{i j}(x)+\right.$ $\left.u_{i j}(x)\right\}$. However, $\lambda\left(\tilde{b}_{i j}(x)+u_{i j}(x)\right) \in \tilde{\Gamma}(x)$, and it follows that $\lambda\left(\tilde{b}_{i j}(x)+\right.$ $\left.v_{i, j}(x)\right) \in \tilde{\Gamma}(x)$, a contradiction.

Proposition 3.3 (Maximum Principle). Assume that $f$ satisfies (1) and (2), $\left(\tilde{b}_{i j}(x)\right)$ is some $n \times n$ continuous symmetric matrix function defined on $\bar{\Omega}$, where $\Omega$ 
is a bounded domain in $\mathbf{R}^{n}$. Let $u$ be a $C^{2}$ function defined on $\Omega$ with $\lambda\left(\tilde{b}_{i j}(x)+\right.$ $\left.u_{i j}(x)\right) \underset{\tilde{\Gamma}}{\in}$ for all $x \in \Gamma$, $v$ be another $C^{2}$ function on $\Omega, \lambda\left(\tilde{b}_{i j}(x)+v_{i j}(x)\right)$ lying outside $\tilde{\tilde{\Gamma}}$, where

$$
\tilde{\tilde{\Gamma}}(x):=\left\{\lambda \in \Gamma \mid f(\lambda)>f\left(\lambda\left(\tilde{b}_{i j}(x)+u_{i j}(x)\right)\right)\right\}
$$

If $u \leqq v$ on $\partial \Omega$, then

$$
u \leqq v \text { in } \Omega
$$

Proof: Let $\varepsilon>0$ be a small positive number; since $\Omega$ is bounded, for $\varepsilon>0$ small enough, $\sqrt{\varepsilon}-\varepsilon|x|^{2} \geqq 0$ for all $x \in \bar{\Omega}$. Let $v^{2}=v-\varepsilon|x|^{2}+\sqrt{\varepsilon}>v, x \in \bar{\Omega}$. Since $\lambda\left(\tilde{b}_{i j}(x)+v_{i j}(x)\right)$ lies outside $\tilde{\tilde{\Gamma}}(x), \lambda\left(\tilde{b}_{i j}(x)+v_{i j}^{e}(x)\right)$ lies outside $\tilde{\Gamma}(x)=\left\{\lambda \in \Gamma \mid f(\lambda) \geqq f\left(\lambda\left(\tilde{b}_{i j}(x)+u_{i j}(x)\right)\right\}\right.$. According to Proposition 3.2, $u \leqq v^{\varepsilon}$ in $\Omega$. Let $\varepsilon \rightarrow 0$, then $u \leqq v$ in $\Omega$.

In what follows we use the method of continuity to prove the existence part.

According to the proof of Proposition 3.1, we can choose two admissable functions $u^{*}, \underline{u}$, such that

$$
\begin{gathered}
\underline{u}=u^{*}=\varphi \text { on } \partial \Omega, \\
\Psi \geqq \psi^{*}+1 \geqq \psi_{1}+2 \text { in } \Omega,
\end{gathered}
$$

where

$$
\begin{aligned}
\underline{\psi} & =f\left(\lambda\left(\underline{u}_{i j}+\tilde{b}_{i j}\right)\right), \\
\psi^{*} & =f\left(\lambda\left(u_{i j}^{*}+\tilde{b}_{i j}\right)\right) .
\end{aligned}
$$

Consider a family of equations:

$$
\begin{aligned}
f\left(\lambda\left(u_{i j}^{\prime}+\tilde{b}_{i j}\right)\right) & =(1-t) \psi^{*}+t \psi=: \psi^{\prime}, \\
\left.u^{\prime}\right|_{\partial \cap} & =\varphi,
\end{aligned}
$$

where $0 \leqq t \leqq 1$. We first derive a priori estimates for the solutions of (55). Since $f\left(\lambda\left(u_{i j}^{\prime}+\tilde{b}_{i j}\right)\right)=\psi^{\prime}<\Psi$, according to Proposition $1.3, u^{\prime} \geqq \underline{u}$ in $\bar{\Omega}$. Let $\bar{u}$ be the solution of

$$
\begin{gathered}
\Delta \bar{u}+\sum_{i} a_{i i}(x)=0, \\
\left.\bar{u}\right|_{\partial \Omega}=\varphi .
\end{gathered}
$$


From (3) we have $\Delta u^{\prime}+\sum_{i} a_{i i}(x) \geqq 0$. According to the maximum principle, $u^{t} \leqq \bar{u}$ in $\bar{\Omega}$. Therefore, we have already obtained the a priori bound for $\left\|u^{\prime}\right\|_{C^{0}(\Omega)}$. In view of the estimate $\underline{u} \leqq u^{\prime} \leqq \bar{u},\left\|D u^{\prime}\right\|_{C^{0}(\partial \Omega)}$ are also bounded. For simplicity, we are going to obtain estimates for $t=1$ in (55), since the same argument works for all $t$.

Let

$$
F\left(u_{i j}+\tilde{b}_{i j}\right)=f\left(\lambda\left(u_{i j}+\tilde{b}_{i j}\right)\right)
$$

consider the linearized operator at $u$,

$$
L w=F_{u_{i j}}\left(u_{i j}+\tilde{b}_{i j}\right) D_{i j}
$$

Differentiating the equation in (55) we see that

$$
\left|L u_{k}\right| \leqq C\left(1+\sum_{i} F_{u_{u}}\right), \quad 1 \leqq k \leqq n .
$$

Using the concavity of $F$, we have

$$
1 \leqq \Psi-\psi=F\left(\underline{u}_{i j}+\tilde{b}_{i j}\right)-F\left(u_{i j}+\tilde{b}_{i j}\right) \leqq L(\underline{u}-u) .
$$

Relations (56) and (57) imply that

$$
L\left(C(\underline{u}-u)+\frac{1}{2} C|x|^{2} \pm u_{k}\right) \geqq C+C \sum_{i} F_{u_{i \prime}} \pm L u_{k} \geqq 0 .
$$

Because of (58) and the fact that $\|D u\|_{C^{0}(a \Omega)}$ is bounded we conclude, using the maximum principle, that $\|D u\|_{C^{\circ}(\bar{\Omega})}$ is also bounded.

We first obtain a priori estimates for the second derivatives of $u$ under the assumption that we already have the bound for $\left\|D_{i j} u\right\|_{C^{0}(\partial \Omega)}$. Let

$$
\partial_{\xi}=\sum_{i} \xi_{i} \partial_{x_{i}}, \quad \xi \in \mathbb{R}^{n}, \quad \sum_{i} \xi_{i}^{2}=1
$$

Differentiate the equation in (55) twice and use the concavity of $F$ (see Section 3 of [7] for the concavity of $F$ ); then we have

$$
L \partial_{\xi}^{2} u \geqq \partial_{\xi}^{2} \psi-C \sum_{i} F_{u_{i t}} \geqq-C-C \sum_{i} F_{u_{i i}} .
$$

Therefore,

$$
L\left(C(\underline{u}-u)+\frac{1}{2} C|x|^{2}+\partial_{\xi}^{2} u\right) \geqq 0 .
$$


Since we assume that $\left\|D^{2} u\right\|_{C^{0}(\partial \Omega)}$ has an a priori bound, we get an a priori upper bound for $\partial_{\xi}^{2} u$ by using the maximum principle, namely, $\max _{\bar{\Omega}} \partial_{\xi}^{2} u \leqq C$.

From the fact that $\Delta u=\partial_{\xi}^{2}+\partial_{\xi_{2}}^{2} u+\cdots+\partial_{\xi_{2}}^{2} u>-C$, where $\left(\xi, \xi_{2}, \cdots, \xi_{n}\right)$ form an orthonormal basis of $\mathbb{R}^{n}$, we see that $\partial_{\xi}^{2} u>-\left\{\partial_{\xi_{2}}^{2} u+\cdots+\partial_{\xi_{n}}^{2}\right\} \geqq$ $-C$. Notice that $\partial_{\xi}=(1 / \sqrt{2})\left(\partial_{i}+\partial_{j}\right), \partial_{\xi}^{2} u=\partial_{i i} u+2 \partial_{i j} u$, where $\xi$ is some unit vector in $\mathbb{R}^{n}$. Combining the above estimates we see that $\left\|\partial_{i j} u\right\|_{C^{0}(\bar{\Omega})}$ is under control.

We now turn to the estimates of the second derivatives of $u$ on the boundary. We may suppose that the boundary point is the origin, that the positive $x_{n}$-axis is the interior normal there, and that the boundary is locally represented by

$$
x_{n}=\rho\left(x^{\prime}\right)=\frac{1}{2} \sum_{1}^{n-1} \kappa_{\alpha} x_{\alpha}^{2}+O\left(\left|x^{\prime}\right|^{3}\right) .
$$

Here and in what follows the Greek letters $\alpha, \beta$ always run from 1 to $n-1$.

At the origin we have, assuming $\varphi$ has been extended smoothly to $\bar{\Omega}$ with $\varphi_{n}(0)=0$, that

$$
u_{\alpha \beta}=\varphi_{\alpha \beta}-u_{n} \rho_{\alpha \beta}, \quad \alpha, \beta<n .
$$

Thus,

$$
\left|u_{\alpha \beta}(0)\right| \leqq C, \quad \alpha, \beta<n .
$$

Differentiating the equation in (55) we see that

$$
\left|L u_{k}\right| \leqq C\left(1+\sum_{i} F_{u_{i i}}\right)
$$

For $i \neq j$, we consider the infintesimal rotation $x_{i} \partial_{j}-x_{j} \partial_{i}$ and see that

$$
\left|L\left(x_{i} \partial_{j}-x_{j} \partial_{i}\right) u\right| \leqq C\left(1+\sum_{i} F_{u_{i}}\right) .
$$

By subtracting a linear function we may suppose that $u=\varphi=0$ and $u_{\beta}=\varphi_{\beta}=0$ at the origin for $\beta<n$. For $\alpha<n$, let

$$
T=\partial_{\alpha}+\kappa_{\alpha}(0)\left(x_{\alpha} \partial_{n}-x_{n} \partial_{\alpha}\right),
$$

so that, on $\partial \Omega$, near the origin,

$$
|T(u-\varphi)| \leqq C\left|x_{n}\right| \leqq C\left|x^{\prime}\right|^{2} .
$$

It follows from (60) and (61) that

$$
|L(T(u-\varphi))| \leqq C\left(1+\sum_{i} F_{u_{i i}}\right) .
$$


We construct a barrier function: According to (54), $\left(\kappa_{1}, \cdots, \kappa_{n-1}, R\right) \in \Gamma$. Choose $\delta>0$ so small that $\left(\kappa_{1}-2 \delta, \cdots, \kappa_{n-1}-2 \delta, R\right) \in \Gamma$. Let

$$
\sigma=\sum_{a} \frac{1}{2}\left(\kappa_{a}-28\right) x_{a}^{2}-x_{n}+\frac{1}{2} R x_{n}^{2}
$$

then, at the origin,

$$
\left(\sigma_{i j}\right)=\operatorname{diag}\left(\kappa_{1}-2 \delta, \cdots, \kappa_{n-1}-2 \delta, R\right) .
$$

Therefore, there exists a compact set $K \subset \Gamma$, such that $\lambda\left(\sigma_{i j}(x)\right) \in K$ for all $x \in B_{\varepsilon}=\{x:|x|<\varepsilon\}$, where $\varepsilon>0$ is small but under control. Clearly, there exists another compact set $\tilde{K} \subset \Gamma$, such that, for $A>0$ large but under control,

$$
\lambda\left(\sigma_{i j}(x)+\frac{1}{A} \tilde{b}_{i j}(x)\right) \in \tilde{K} \quad \text { for all } \quad x \in B_{\varepsilon} .
$$

Using the concavity of $F$, we have

$$
\begin{aligned}
L(A \sigma-u) & \geqq F\left(A \sigma_{i j}+\tilde{b}_{i j}\right)-F\left(u_{i j}+\tilde{b}_{i j}\right) \\
& =F\left(A\left(\sigma_{i j}+\frac{1}{A} \tilde{b}_{i j}\right)\right)-\psi .
\end{aligned}
$$

Therefore, using (53), we can make $L(A \sigma-u)$ as large as we want by choosing $A$ to be large.

Let $v=\frac{1}{2} \delta^{2}|x|^{2}$; we use $A(\sigma+v)-u$ as a barrier function, where $A$ is chosen to be very large. We are going to apply the maximum principle in $B_{e} \cap \Omega$. The following estimates hold when we take $\delta, \varepsilon>0$ to be small enough and $A>0$ to be large enough:

(i) On $\partial \Omega \cap B_{e}$,

$$
\begin{aligned}
\sigma & =-\delta\left|x^{\prime}\right|^{2}+\frac{1}{2} R\left|x_{n}\right|^{2} \leqq-\frac{1}{2} \delta\left|x^{\prime}\right|^{2}, \\
\sigma+v & \leqq-\frac{1}{2} \delta\left|x^{\prime}\right|^{2}+\frac{1}{2} \delta^{2}|x|^{2} \leqq-\frac{1}{4} \delta\left|x^{\prime}\right|^{2}, \\
|u| & \leqq C\left|x^{\prime}\right|^{2} .
\end{aligned}
$$

Thus

$$
A(\sigma+v)-u \leqq-\frac{1}{4} \delta A\left|x^{\prime}\right|^{2}+C\left|x^{\prime}\right|^{2} \leqq-\frac{1}{8} \delta A\left|x^{\prime}\right|^{2}
$$

(ii) On $\partial B_{e} \cap \Omega$ with $x_{n} \leqq 0$,

$$
\begin{array}{r}
\frac{1}{2} \sum_{\alpha} \kappa_{\alpha} x_{\alpha}^{2}-x_{n} \leqq 0, \\
x_{n} \leqq 0 .
\end{array}
$$


It follows that

$$
\left|x_{n}\right|=-x_{n} \leqq-\frac{1}{2} \sum_{\alpha} \kappa_{\alpha} x_{\alpha}^{2} \leqq C\left|x^{\prime}\right|^{2}
$$

Using the fact that $\left(x^{\prime}, x_{n}\right) \in \partial B_{e}$, we can also obtain that

$$
\begin{aligned}
\varepsilon^{2} & =x_{n}^{2}+\left|x^{\prime}\right|^{2} \leqq C^{2}\left|x^{\prime}\right|^{4}+\left|x^{\prime}\right|^{2} \leqq 2\left|x^{\prime}\right|^{2}, \\
\left|x^{\prime}\right| & \geqq \frac{1}{2} \varepsilon .
\end{aligned}
$$

Thus

$$
\begin{aligned}
\sigma & \leqq-\delta\left|x^{\prime}\right|^{2}+\frac{1}{2} R x_{n}^{2} \leqq-\frac{1}{2} \delta\left|x^{\prime}\right|^{2} \\
|u| & \leqq C\left|x_{n}\right|+|\varphi| \leqq C\left|x^{\prime}\right|^{2} \\
A(\sigma+v)-u & \leqq-\frac{1}{2} A \delta\left|x^{\prime}\right|^{2}+\frac{1}{2} A \delta^{2}|x|^{2}-C\left|x^{\prime}\right|^{2} \\
& \leqq-\frac{1}{4} A \delta\left|x^{\prime}\right|^{2} \leqq-\frac{1}{16} A \delta \varepsilon^{2}
\end{aligned}
$$

(iii) On $\partial B_{\varepsilon} \cap \Omega$ with $x_{n} \geqq 0$ and $\frac{1}{2} \Sigma_{\alpha}\left(1+\left|\kappa_{\alpha}\right|\right) x_{\alpha}^{2}-\frac{1}{2} x_{n} \leqq 0$,

$$
\begin{aligned}
\sigma & \leqq-\frac{1}{2} x_{n}-\delta\left|x^{\prime}\right|^{2}+\frac{1}{2} R x_{n}^{2} \leqq-\frac{1}{4} x_{n}, \\
|u| & \leqq C x_{n}+C|\varphi| \leqq C x_{n}+C\left|x^{\prime}\right|^{2} \leqq C x_{n}, \\
\varepsilon^{2} & =x_{n}^{2}+\left|x^{\prime}\right|^{2} \leqq C x_{n}, \\
A(\sigma+v)-u & \leqq-\frac{1}{4} A x_{n}+\frac{1}{2} A \delta^{2}|x|^{2}-C x_{n} \\
& \leqq-\frac{1}{8} A x_{n} \leqq-A \varepsilon^{2} / 8 C .
\end{aligned}
$$

(iv) On $\partial B_{e} \cap \Omega$ with $x_{n} \geqq 0, \frac{1}{2} \Sigma_{\alpha}\left(1+\left|\kappa_{\alpha}\right|\right) x_{\alpha}^{2}-\frac{1}{2} x_{n} \geqq 0$,

$$
\begin{aligned}
\sigma & \leqq-\delta\left|x^{\prime}\right|^{2}+\frac{1}{2} R x_{n}^{2} \leqq-\frac{1}{2} \delta\left|x^{\prime}\right|^{2}, \\
|u| & \leqq C\left|x^{\prime}\right|^{2}, \\
\varepsilon^{2} & =x_{n}^{2}+\left|x^{\prime}\right|^{2} \leqq C\left|x^{\prime}\right|^{4}+\left|x^{\prime}\right|^{2} \leqq 2\left|x^{\prime}\right|^{2}, \\
A(a+v)-u & \leqq-\frac{1}{2} A \delta\left|x^{\prime}\right|^{2}+\frac{1}{2} A \delta^{2}|x|^{2}-C\left|x^{\prime}\right|^{2} \\
& \leqq-\frac{1}{4} A \delta\left|x^{\prime}\right|^{2} \leqq-\frac{1}{8} A \delta \varepsilon^{2} .
\end{aligned}
$$


Using the estimates above, we may choose $A>0$ so large, but under control, that

$$
\begin{gathered}
A(\sigma+v)-u \leqq \pm T(u-\varphi) \text { on } \partial\left(B_{\varepsilon} \cap \Omega\right) \\
L(A(\sigma+v)-u \pm T(u-\varphi)) \geqq 0
\end{gathered}
$$

According to the maximum principle we have

$$
A(\sigma+v)-u \leqq \pm T(u-\varphi) \text { in } \Omega \cap B_{\varepsilon} .
$$

which implies that

$$
\left|\frac{\partial}{\partial x_{n}}(T(u-\varphi))\right|_{x=0} \leqq\left|\frac{\partial}{\partial x_{n}}(A(\sigma+v)-u)\right|_{x=0} \leqq C .
$$

Therefore,

$$
\left|u_{n \alpha}(0)\right| \leqq C, \quad \alpha<n .
$$

Finally we need to estimate $u_{n n}(0)$. At the origin, $\Sigma_{\alpha<n}\left(u_{\alpha \alpha}+u_{n n}\right)>-C$ according to (3) which implies that $u_{n n}(0) \geqq-C$. Therefore, we only have to prove that

$$
m=u_{n n}(0) \leqq C
$$

When $\Gamma$ is of type 2 , since $\left|u_{\alpha \beta}(0)\right| \leqq C, \alpha, \beta<n$, we have at the origin that

$$
\left|\lambda_{\alpha}\right| \leqq C \text { for } \alpha<n
$$

Since $\Gamma$ is of type 2 , it follows that for some constant $M$, depending only on $C$ and $\Gamma,\left(\lambda_{1}, \cdots, \lambda_{n-1}, M\right)$ lies in a compact subset of $\Gamma$. Using condition (52) we infer that, at the origin, $\lambda_{n}$ is bounded from above. Hence so is $u_{n n}(0)$ and (62) is proved in this case.

In the following we assume that $\Gamma$ is of type 1 . Without loss of generality, let us assume that $\tilde{b}_{i j}(0)=0$. Let $\Gamma^{\prime}$ denote the projection to $\lambda^{\prime}=\left(\lambda_{1}, \cdots, \lambda_{n-1}\right)$ of $\Gamma$. Since $\Gamma$ is of type $1, \Gamma^{\prime}$ is an open convex cone in $\mathbb{R}^{n-1}$ which is not all of $\mathbb{R}^{n-1}$. At the origin, $u=\varphi=0, u_{\beta}=\varphi_{\beta}=0$. (From now on derivatives are computed at the origin.) Using condition (54) we see that, for large positive and negative $t$, the eigenvalues $\lambda^{\prime}$ of the $(n-1) \times(n-1)$ matrix $\left\{\varphi_{\alpha \beta}(0)+t \rho_{\alpha \beta}(0)\right\}$ belong to $\Gamma^{\prime}$ and the complement of $\bar{\Gamma}^{\prime}$, respectively. Let $t_{0}$ be the first value of $t$ as we decrease $t$ from $+\infty$ such that

$$
\lambda^{\prime}\left(\sigma_{\alpha \beta}\right) \in \partial \Gamma^{\prime}
$$

where

$$
\sigma=\frac{1}{2}\left(\varphi_{\alpha \beta}(0)+t_{0} \rho_{\alpha \beta}(0)\right) x_{\alpha} x_{\beta}
$$


Then $\left|t_{0}\right| \leqq C$, for $C$ under control. Without loss of generality we may suppose that $\sigma_{\alpha \beta}=\tilde{\lambda}_{\alpha} \delta_{\alpha \beta}$, where $\tilde{\lambda}_{1} \leqq \cdots \leqq \tilde{\lambda}_{n-1}$.

Suppose that $m$ is very large. By Lemma 1.2 in [7] the first $n-1$ eigenvalues of $u_{i j}(0), \lambda^{\prime}\left(u_{i j}\right)$ are given by

$$
\lambda^{\prime}\left(u_{\alpha \beta}\right)+o(1)
$$

Our aim is to establish the estimate

$$
-u_{n}(0) \geqq t_{0}+\eta,
$$

for some fixed $\eta>0$. It then follows that $\lambda^{\prime}\left(u_{\alpha \beta}\right)$ is in $\Gamma^{\prime}$ and its distance to $\partial \Gamma^{\prime}$ is greater than some positive constants $\eta^{\prime}$. If $m$ is very large, $\lambda^{\prime}\left(u_{i j}\right)$ is close to $\lambda^{\prime}\left(u_{\alpha \beta}\right)$ and so its distance to $\partial \Gamma^{\prime}$ is greater than $\frac{1}{2} \eta^{\prime}$. Thus, for some constant $M$ under control, $\left(\lambda^{\prime}\left(u_{i j}\right), M\right)$ belongs to a compact set in $\Gamma$. But condition (53) yields a bound on $\lambda_{n}$, hence on $m=u_{n n}(0)$.

On $\partial \Omega$ near the origin, we have

$$
u=\varphi=\frac{1}{2} \sum_{\alpha, \beta} \varphi_{\alpha \beta}(0) x_{\alpha} x_{\beta}+t_{0}\left(\frac{1}{2} \rho_{\alpha \beta}(0) x_{\alpha} x_{\beta}-\rho\left(x^{\prime}\right)\right)+P\left(x^{\prime}\right)+R\left(x^{\prime}\right),
$$

where $P$ is a homogeneous cubic polynomial in $x^{\prime}$ and

$$
|R| \leqq C\left|x^{\prime}\right|^{4}
$$

As explained in [7] (see Lemma 6.1 in [7]), at $\lambda^{\prime}\left(\sigma_{\alpha \beta}\right)=\tilde{\lambda}$, the cone has a plane of support, namely, there exists $\mu_{1} \geqq \cdots \geqq \mu_{n-1} \geqq 0$ such that

$$
\sum \mu_{\alpha}=1, \quad \sum \mu_{\alpha} \tilde{\lambda}_{\alpha}=0, \quad \Gamma^{\prime} \text { lies in }\left\{\sum \mu_{\alpha} \lambda_{\alpha}>0\right\}
$$

Let $S$ be a surface represented locally by

$$
x_{n}=\rho\left(x^{\prime}\right)-\frac{1}{2} \tau\left|x^{\prime}\right|^{2}
$$

with $0<\tau$ small and let $d(x)$ denote the distance of $x \in \Omega$ from $S$. At the origin the eigenvalues of $d_{i j}$ are (not in order)

$$
\lambda\left(d_{i j}\right)=\left(\tau-\kappa_{1}, \cdots, \tau-\kappa_{n-1}, 0\right) .
$$

By the hypotheses $(54),\left(\kappa_{1}, \cdots, \kappa_{n-1}\right) \in \Gamma^{\prime}$ and hence for fixed positive $\tau$ sufficiently small we have

$$
\sum \mu_{\alpha} \kappa_{\alpha}-\tau \geqq \alpha>0
$$

for some fixed constant $a$ (independent of the particular point on $\partial \Omega$ which we 
have chosen as origin). This holds for any ordering of $\kappa_{\alpha}$ and we take $\tau$ to be so fixed from now on.

For $\alpha=1, \cdots, n-1$, let $b^{\alpha}(x)$ be smooth orthonormal vector fields in $\bar{\Omega}$ near 0 tangent to the level surface $d=$ constant and such that $b^{\alpha}\left(0, x_{n}\right)$ is the unit vector in the $x_{\alpha}$ direction. Set

$$
a^{\alpha}(x)=\sqrt{\mu_{\alpha}} b^{\alpha}(x)
$$

$$
\alpha=1, \cdots, n-1
$$

$$
\Lambda=\sum_{i, j} \sum_{\alpha} a_{i}^{\alpha} a_{j}^{\alpha} \partial_{i} \partial_{j}
$$

Let

$$
D_{h}=\left\{x \in \Omega:\left|x^{\prime}\right|<h, x_{n}<h^{2}\right\}
$$

in $D_{h}$, where for $h \leqq h_{0}$ small,

$$
d \leqq C h^{2}
$$

we shall employ the barrier function

$$
v=w+\eta\left(C\left|x^{\prime}\right|^{2}-x_{n}\right)
$$

where

$$
w=\sigma\left(x^{\prime}\right)-t_{0} x_{n}+P\left(x^{\prime}\right)+l\left(x^{\prime}\right)\left(\frac{1}{2} \tau\left|x^{\prime}\right|^{2}-d\right)+\frac{1}{2} M d^{2} .
$$

Here $C$ is fixed so that $C\left|x^{\prime}\right|^{2}-x_{n} \geqq 0$ on $\partial \Omega \cap D_{h_{0}} ; l\left(x^{\prime}\right)$ is a suitable linear function of $x^{\prime}, M$ and $\eta$ will be chosen, respectively, as large and small positive constants, so that we will have

$$
u \leqq v \text { on } \partial D_{h},
$$

and so that, at every point in $D_{h}$,

$$
\lambda\left(v_{i j}+\tilde{b}_{i j}\right) \text { is not in } \tilde{\Gamma},
$$

where $\tilde{\Gamma}=\left\{\lambda \in \Gamma: f(\lambda) \geqq \psi_{0}\right\}$. It then follows from Proposition 1.3 that

$$
u \leqq v \text { in } D_{h},
$$

which yields (63).

Let $\hat{m}\left(x^{\prime}\right)$ be a linear function, such that

$$
\hat{m}\left(x^{\prime}\right)+O\left(\left|x^{\prime}\right|^{2}\right)+O\left(\left|x_{n}\right|\right)=\sum_{i, j} \sum_{\alpha} a_{i}^{\alpha} a_{j}^{\alpha} \tilde{b}_{i j}(x) .
$$


It is not difficult to see that the coefficients of $\hat{\boldsymbol{m}}\left(x^{\prime}\right)$ are under control. As proved in [7] (see Lemma 6.2 in [7]), $\lambda\left(v_{i j}(x)+\tilde{b}_{i j}(x)\right.$ ) does not belong to $\Gamma$ if

$$
\sum_{i, j} \sum_{\alpha} a_{i}^{a} a_{j}^{\alpha}\left(v_{i j}(x)+\tilde{b}_{i j}(x)\right)<0
$$

which is equivalent to

$$
\Lambda w<-\hat{m}\left(x^{\prime}\right)+O\left(\left|x^{\prime}\right|^{2}\right)+O\left(\left|x_{n}\right|\right) .
$$

At the origin,

$$
-\Lambda d=-\sum d_{i j} a_{i}^{\alpha} a_{j}^{\alpha}=\sum \mu_{\alpha} \kappa_{\alpha}-\tau \geqq a>0 .
$$

Thus near the origin we have

$$
-\Lambda d \geqq \frac{1}{2} a>0 .
$$

At $\left(0, x_{n}\right), \Lambda \sigma=0$ because of (58), so near the origin,

$$
\Lambda \sigma=m_{1}\left(x^{\prime}\right)+O\left(|x|^{2}\right),
$$

where $m_{1}\left(x^{\prime}\right)$ is a linear function in $x^{\prime}$. It is clear that

$$
\Lambda P=m_{2}\left(x^{\prime}\right)+O\left(|x|^{2}\right),
$$

where $m_{2}\left(x^{\prime}\right)$ is some linear function in $x^{\prime}$.

Let

$$
m\left(x^{\prime}\right)=m_{1}\left(x^{\prime}\right)+m_{2}\left(x^{\prime}\right)+\hat{m}\left(x^{\prime}\right) ;
$$

using Lemma 6.3 in [7], we know that there exists a unique linear function in $x^{\prime}, l\left(x^{\prime}\right)$, such that

$$
\left|\Lambda\left(I\left(x^{\prime}\right)\left(d-\frac{1}{2} \tau\left|x^{\prime}\right|^{2}\right)\right)-m\left(x^{\prime}\right)\right| \leqq C|x|^{2}
$$

where the coefficients of $l\left(x^{\prime}\right)$ are bounded by $C$, which is under control. Noticing the fact that $a^{a}$ is perpendicular to $\nabla d$, we have

$$
\begin{aligned}
\Lambda w= & \left\{\Lambda(\sigma+P)-\left(m_{1}+m_{2}\right)\right\}+\left\{\Lambda\left(l\left(x^{\prime}\right)\left(\frac{1}{2} \tau\left|x^{\prime}\right|^{2}-d\right)\right)+m\left(x^{\prime}\right)\right\} \\
& -\hat{m}\left(x^{\prime}\right)+M d \Lambda d \\
\leqq & O\left(|x|^{2}\right)-\frac{1}{2} M a d-\hat{m} .
\end{aligned}
$$

Clearly, for $x$ near the origin,

$$
d \geqq \frac{1}{C}\left(\left|x_{n}\right|+\left|x^{\prime}\right|^{2}\right),
$$


where $C>0$ is some positive constant under control. Therefore we see that, near the origin,

$$
\Lambda w<-\sum_{i, j} a_{i}^{\alpha} a_{j}^{\alpha} \tilde{b}_{i j}(x)
$$

Hence $\lambda\left(w_{i j}(x)+\tilde{b}_{i j}(x)\right)$ does not belong to $\bar{\Gamma}$ for $x \in \bar{\Omega}$. By the continuity of $w_{i j},\left\{\lambda\left(w_{i,}(x)+b_{i j}(x)\right): x \in \bar{\Omega}\right\}$ is a compact set with a positive distance from $\bar{\Gamma}$.

Let $\eta>0$ be very small; then $\lambda\left(v_{i j}(x)+\tilde{b}_{i j}(x)\right)$ does not belong to $\{\lambda \in \Gamma$ : $f(\lambda) \geqq \psi\}$ for any $x \in \bar{\Omega}$.

On $\partial \Omega \cap D_{h}$ we have, for $h$ small,

$$
\left.\left|\frac{1}{2} \tau\right| x^{\prime}\right|^{2}-\left.d|\leqq C| x^{\prime}\right|^{3} .
$$

Using (64), (67) and (70) we see that

$$
u-w \leqq C\left|x^{\prime}\right|^{4}-\frac{1}{2} M d^{2} .
$$

It follows from (68) and (71) that, when we choose $M$ large,

$$
u-v \leqq u-w \leqq 0 \quad \text { on } \quad \partial \Omega \cap D_{h} .
$$

On $\Omega \cap \partial D_{h}, d \geqq c h^{2} ;$ thus we only need to choose $M$ large in order to have

$$
u-v \leqq 0 \text { on } \Omega \cap \partial D_{h} .
$$

So far we have derived a priori estimates up to second derivatives for solutions $u^{\prime}$ of (55). Since $\left|u^{\prime}\right|_{c^{2}}$ is under control, the linearized operator $L=\sum F_{u_{i j}}\left(\tilde{b}_{i j}+u_{i j}\right) \partial_{i j}$ is a uniformly elliptic operator. It is shown in [7] and [17] that we can derive the estimate

$$
\left|u^{\prime}\right|_{C^{2, \mu}(\bar{\Omega})} \leqq C,
$$

for some $0<\mu<1$. Therefore, we can use the method of continuity to conclude the existence part of Theorem 3.1 .

\section{More General Equations}

For

$$
\begin{gathered}
\operatorname{det}\left(u_{i j}\right)= \\
\psi(x, u, \nabla u) \text { in } \Omega, \\
\left.u\right|_{\partial \Omega}=\varphi,
\end{gathered}
$$

where $\Omega$ is a smooth bounded convex domain, $\psi>0, \varphi$ are sufficiently smooth functions. 
It has been proved in [5] that if we assume the existence of a subsolution, namely, that there exists $\underline{u} \in C^{2}(\bar{\Omega})$ with $\left.\underline{u}\right|_{\partial \Omega}=\varphi$ and

$$
\operatorname{det}\left(\underline{u}_{i j}\right) \geqq \psi(x, \underline{u}, \nabla \underline{u}) \text { in } \Omega,
$$

then (72) has a strictly convex solution $u \in C^{\infty}(\bar{\Omega})$ with $u \geqq \underline{u}$. If $\psi_{u} \geqq 0$, this solution is unique.

The above result is proved by using Leray-Schauder degree theory. As usual the crucial step is to make a priori estimates for solutions of the equation.

A natural question is whether we can prove a similar result for more general equations, which have been studied in [7], [8] and the previous sections. The result we present in this section permits the dependence of $\psi$ on $u$, but not on $\nabla u$.

Let us consider

$$
\begin{gathered}
f\left(\lambda\left(\tilde{a}_{i j}(x)+u_{i j}(x)\right)\right)=\psi(x, u), \\
\left.u\right|_{\partial \Omega}=\varphi .
\end{gathered}
$$

Definition 4.1. $\underline{u} \in C^{2}(\bar{\Omega})$ is said to be a subsolution of (73) if $\underline{u}$ satisfies

$$
\begin{aligned}
f\left(\lambda\left(\tilde{a}_{i j}(x)+\underline{u}_{i j}(x)\right)\right) & \geqq \psi(x, \underline{u}(x)), \quad x \in \bar{\Omega}, \\
\lambda\left(\tilde{a}_{i j}(x)+\underline{u}_{i j}(x)\right) & \in \Gamma \text { for all } x \in \bar{\Omega}, \\
\left.u\right|_{\partial \Omega} & =\varphi .
\end{aligned}
$$

Our main result in this section is the following theorem.

TheOREM 4.1. Suppose that $f, \Omega$ satisfy (1), (2), (5), (52), (53), (54) and

$$
\lim _{\lambda \rightarrow \lambda_{0}} \sup f(\lambda) \leqq 0 \quad \text { for all } \lambda_{0} \in \partial \Gamma
$$

Assume that $\psi>0,\left(\tilde{a}_{i j}\right), \varphi$ are sufficiently smooth functions and also that (73) has a subsolution. Then (73) has a solution $u \in C^{\infty}(\bar{\Omega})$ with $\lambda\left(\tilde{a}_{i j}(x)+u_{i j}(x)\right) \in \Gamma$ for all $x \in \bar{\Omega}, u \geqq \underline{u}$. If $\psi_{u} \geqq 0$, this solution is unique.

Without loss of generality we can always assume that $\left(\tilde{a}_{i j}\right)$ is positive definite, namely, that there exist two positive numbers $\mu, \nu$, such that, as matrices,

$$
\mu I \leqq\left(\tilde{a}_{i j}(x)\right) \leqq \nu I \text { for all } x \in \bar{\Omega} .
$$

The reason is that we can choose a very large number $\Lambda$, such that $\Lambda I+\left(\tilde{a}_{i j}\right)$ is positive definite; we then let $u=\Lambda|x|^{2}+v$ and solve the equation for $v$.

The uniqueness part of Theorem 4.1 follows from Proposition 3.2. In order to prove the existence part of Theorem 4.1 we first obtain a priori estimates for 
solutions of (73) with $\lambda\left(\tilde{a}_{i j}(x)+u_{i j}(x)\right) \in \Gamma$ for all $x \in \bar{\Omega}$ :

$$
|u|_{C^{5}(\bar{\Omega})} \leqq C .
$$

Using the above estimate, we can apply Leray-Schauder degree theory to prove the existence of solutions to (73).

If $\underline{u}$ is a solution, we have nothing to prove; therefore we always assume that $\underline{u}$ is not a solution. Let $u^{0}$ be a $C^{\infty}(\bar{\Omega})$ function with $\lambda\left(\tilde{a}_{i j}(x)+u_{i j}^{0}(x)\right) \in \Gamma$, $\bar{x} \in \bar{\Omega},\left.u^{0}\right|_{\partial \Omega}=\varphi$ and satisfy

$$
f\left(\lambda\left(\tilde{a}_{i j}(x)+u_{i j}^{0}(x)\right)\right)<f\left(\lambda\left(\tilde{a}_{i j}(x)+\underline{u}_{i j}(x)\right)\right)
$$

By Theorem 3.1, many such functions exist.

According to the maximum principle and the Hopf lemma we also have

$$
\underline{u}<u^{0} \text { in } \Omega \text { and } \partial_{\nu}\left(\underline{u}-u^{0}\right)>0 \text { on } \partial \Omega \text {, }
$$

where $\partial_{v}$ is exterior normal differentiation.

For $0 \leqq t \leqq 1$, consider

$$
\begin{gathered}
f\left(\lambda\left(\tilde{a}_{i j}(x)+u_{i j}(x)\right)\right)=t \psi(x, u)+(1-t) f\left(\lambda\left(\tilde{a}_{i j}(x)+u_{i j}^{0}(x)\right)\right) \\
\left.u\right|_{\partial \Omega}=\varphi
\end{gathered}
$$

In the space $C_{0}^{5}(\bar{\Omega})$, we consider the cone

$$
C:=\left\{v \in C_{0}^{5}(\bar{\Omega}) \mid v>0 \text { in } \Omega,\left.v\right|_{\partial \Omega}=0,\left.v_{\nu}\right|_{\partial \Omega}<0\right\}
$$

Here $C_{0}^{S}(\bar{\Omega})$ is the space of functions in $C^{5}$ which vanish on $\partial \Omega$.

For $R$ large we shall use degree theory to find a solution $u$ of (73) with $\lambda\left(\tilde{a}_{i j}(x)+u_{i j}(x)\right) \in \Gamma, x \in \bar{\Omega}$ :

$$
u=\underline{u}+v, \quad v \in C_{R},
$$

where $C_{R}=C \cap\left\{\left.v|| v\right|_{5}<R\right\} \cap\left\{v \mid \lambda\left(\tilde{a}_{i j}+u_{i j}\right) \in \Gamma\right\}$. First choose $R$ so large that $\left|u^{0}-\underline{u}\right|_{S}<R$ and so that, by (75), there is no solution of (73) given by (77) with $|v|_{s}=R$. Next we claim that there is no solution $u=\underline{u}+v$ with $v$ on the remaining part of the boundary of $C_{R}$. For if $u$ is such a solution, we would have $u \geqq \underline{u}$ and one of the following would occur:

(i) $\lambda\left(\tilde{a}_{i j}+u_{i j}\right) \in \partial \Gamma$ at some point in $\bar{\Omega}$.

(ii) $u=\underline{u}$ at some point in $\Omega$ or $(u-\underline{u})_{v}\left(x_{0}\right)=0$ at some $x_{0} \in \partial \Omega$. 
Because of the positivity of $\psi$ and condition (74), (i) never occurs. If (ii) occurred, we must have $t>0$. But then

$$
\begin{aligned}
& f\left(\lambda\left(\tilde{a}_{i j}+u_{i j}\right)\right)-f\left(\lambda\left(\tilde{a}_{i j}+\underline{u}_{i j}\right)\right)-t(\psi(x, u)-\psi(x, \underline{u})) \\
& =-t\left(f\left(\lambda\left(\tilde{a}_{i j}+\underline{u}_{i j}\right)\right)-\psi(x, \underline{u})\right) \\
& \quad+(1-t)\left(f\left(\lambda\left(\tilde{a}_{i j}+u_{i j}^{0}\right)\right)-f\left(\lambda\left(\tilde{a}_{i j}+\underline{u}_{i j}\right)\right)\right) \\
& \leqq 0,
\end{aligned}
$$

with strict inequality holding somewhere.

Using the maximum principle and the Hopf lemma in generalized form (see [12] and [19]), we see that $u \equiv \underline{u}$, contradicting the fact that we have strict inequality somewhere in (78).

Now for $v \in C_{R}$ we define a map

$$
v \mapsto u^{t},
$$

where $\boldsymbol{u}^{t}$ is the unique solution of

$$
\begin{aligned}
& f\left(\lambda\left(\tilde{a}_{i j}+u_{i j}^{t}\right)\right)=t \psi(x, \underline{u}+v)+(1-t) f\left(\lambda\left(\tilde{a}_{i j}+u_{i j}^{0}\right)\right) \text { in } \Omega, \\
& \left.u^{t}\right|_{\partial \Omega}=\varphi, \\
& \lambda\left(\tilde{a}_{i j}(x)+u_{i j}^{i}(x)\right) \in \Gamma, \\
& x \in \bar{\Omega},
\end{aligned}
$$

given by Theorem 3.1. Note that for $t=0$, this solution is $u^{0}$. Set

$$
v^{t}=u^{t}-\underline{u}=: T, v \text {. }
$$

By elliptic theory, $T$, is a continuous map in $C^{S}$ and in fact

$$
\left|u^{t}\right|_{5+\mu} \leqq K \text { is independent of } t,
$$

for $0<\mu<1$. Consequently, $T_{t}$ is a compact map. We have shown that there are no solutions of

$$
v-T, v=0
$$

on $\partial C_{R}$. Thus the degree

$$
\operatorname{deg}\left(I-T_{i}, C_{R}, 0\right)=\gamma
$$

is well defined and independent of $t$. For $t=0$, the only solution of (76) is $u^{0}=\underline{u}+v^{0}$ with $v^{0} \in C_{R}$. Therefore, the linearized operator of $f\left(\lambda\left(\tilde{a}_{i j}+u_{i j}\right)\right)$ at $u^{0}$ is invertible. It follows easily that $v^{0}$ is a regular point of $I-T_{0}$, i.e., that the linearized operator $I-T_{0}^{\prime}\left(v^{0}\right)$ is invertible. Consequently, $\gamma= \pm 1$, and (76) has a solution $v^{t}$ in $C_{R}$ for all $0 \leqq t \leqq 1$. Then the function $u^{1}=\underline{u}+v^{1}$ is a solution of (73). 
To establish the estimates (75) we shall consider only the case $t=1$, since the same arguments hold for any $t$. Furthermore, as remarked before, we see that we only need to obtain estimates for derivatives of solutions up to second order.

Let $\bar{u}$ be the solution of

$$
\begin{gathered}
\Delta \bar{u}+\sum_{i} a_{i i}(x)=0 \text { in } \Omega, \\
\left.\bar{u}\right|_{\partial \Omega}=\varphi .
\end{gathered}
$$

By (3) and the maximum principle,

$$
\underline{u} \leqq u \leqq \bar{u} \text { in } \bar{\Omega} .
$$

Therefore we now have estimates for $|u|_{C^{0}(\bar{\Omega})}$ and $|\nabla u|_{C^{0}(\partial \Omega)}$. Because of the $C^{0}$ estimate we have just obtained and the positivity of $\psi$, there exists some positive number $\delta_{0}$, such that

$$
\psi(x, u)>\delta_{0}>0
$$

The interior gradient estimate follows from an argument similar to that in the proof of Theorem 1.1. The boundary estimate for the second derivatives can be obtained as in the proof of Theorem 3.1, and the interior second derivative estimate follows also from an argument similar to that in the proof of Theorem 1.1. This completes the proof.

\section{Appendix: Some Properties of Symmetric Functions}

Let

$$
f(\lambda)=\sum_{i_{1}<\cdots<i_{k}} \lambda_{i_{1}} \cdots \lambda_{i_{k}}>0, \quad \lambda \in \mathbf{R}^{n}, 1 \leqq k \leqq n
$$

let $\Gamma$ be the connected component of $\left\{\lambda \in \mathbf{R}^{n}: f(\lambda)>0\right\}$ containing $\left\{\lambda \in \mathbf{R}^{n}\right.$ : $\left.\lambda_{1}>0, \cdots, \lambda_{n}>0\right\}$. We know from [7] that

$$
f_{\lambda_{1}}>0, \quad f_{\lambda_{i_{1}} \lambda_{i_{2}}}>0, \cdots, f_{\lambda_{i_{1}} \cdots \lambda_{i_{h-1}}}>0
$$

for all $i_{1}, \cdots, i_{k-1}($ distinct), all $\lambda \in \Gamma$.

Lemma A.1. Let $1 \leqq k \leqq n, \lambda \in \Gamma$. Suppose that

$$
\lambda_{1} \geqq \cdots \geqq \lambda_{k} \geqq \cdots \geqq \lambda_{n},
$$

Then

$$
\lambda_{k}>0 \text { and } f(\lambda) \leqq\left(\begin{array}{l}
n \\
k
\end{array}\right) \lambda_{1} \cdots \lambda_{k} .
$$


Proof: We have

$$
\begin{aligned}
& f(\lambda)=\frac{1}{k} \sum_{i} \lambda_{i} f_{\lambda_{i}}(\lambda) \\
& \leqq \frac{1}{k} \sum_{\lambda_{i_{1}}>0} \lambda_{i_{1}} f_{\lambda_{i_{1}}}(\lambda) \\
& =\frac{1}{k} \sum_{\lambda_{i_{1}}>0} \lambda_{i_{1}} \frac{1}{k-1} \sum_{\lambda_{i_{2}}} \lambda_{i_{2}} f_{\lambda_{i_{1}} \lambda_{i_{2}}}(\lambda) \\
& \leqq \frac{1}{k(k-1)} \sum_{\lambda_{i_{1}}, \lambda_{i_{2}}>0} \lambda_{i_{1}} \lambda_{i_{2}} f_{\lambda_{i_{1}} \lambda_{i_{2}}}(\lambda) \\
& \leqq \frac{1}{k !} \sum_{\lambda_{i_{1}}>0, \cdots, \lambda_{i_{4}}>0} \lambda_{i_{1}} \cdots \lambda_{i_{k}} f_{\lambda_{i_{1}} \cdots \lambda_{i_{k}}}(\lambda) \\
& =\sum_{\substack{\lambda_{i_{1}}>0, \cdots, \lambda_{i_{k}}>0 \\
i_{1}<\cdots<i_{k}}} \lambda_{i_{1}} \cdots \lambda_{i_{k}} .
\end{aligned}
$$

Hence $\lambda_{k}>0$ and

$$
f(\lambda) \leqq\left(\begin{array}{l}
n \\
k
\end{array}\right) \lambda_{1} \cdots \lambda_{k}
$$

Lemma A.2. Suppose $1 \leqq k \leqq n, \lambda \in \Gamma, \lambda_{1} \geqq \lambda_{2} \geqq \cdots \geqq \lambda_{n}$; then

$$
f_{\lambda_{n}} \geqq \cdots \geqq f_{\lambda_{1}} \text {. }
$$

Proof: For $l=1, \cdots, n-1$,

$$
\begin{aligned}
& f_{\lambda_{1+1}}=\sum_{\substack{i_{1}<\ldots<i_{k-1} \\
i_{1} \neq 1+1}} \lambda_{i_{1}} \cdots \lambda_{i_{k-1}} \\
& =\lambda_{1} \sum_{\substack{i_{1}<\ldots<i_{k-2} \\
i_{s} \neq 1+1,1}} \lambda_{i_{1}} \cdots \lambda_{i_{k-2}}+\sum_{\substack{i_{1}<\ldots<i_{k-1} \\
i_{s} \neq 1+1,1}} \lambda_{i_{1}} \cdots \lambda_{i_{k-1}} \\
& =\left(\lambda_{l}-\lambda_{l+1}\right) \sum_{\substack{i_{1}<\ldots<i_{k-2} \\
i_{s} \neq l+1, l}} \lambda_{i_{1}} \cdots \lambda_{i_{k-2}}+\lambda_{l+1} \sum_{\substack{i_{1}<\ldots<i_{k-2} \\
i_{s} \neq l+1, l}} \lambda_{i_{1}} \cdots \lambda_{i_{k-2}} \\
& +\sum_{\substack{i_{1}<\ldots<i_{k-1} \\
i_{x} \neq l+1.1}} \lambda_{i_{1}} \cdots \lambda_{i_{k-1}} \\
& =\left(\lambda_{l}-\lambda_{l+1}\right) f_{\lambda_{l+1} \lambda_{l}}+f_{\lambda_{l}} \\
& \geqq f_{\lambda_{l}} \text {. }
\end{aligned}
$$


Lemma A.3. Suppose $\lambda \in \Gamma, 2 \leqq k \leqq n$, and

$$
\lambda_{1} \geqq \cdots \geqq \lambda_{n} \text {. }
$$

Then, for $k \leqq s \leqq n$,

$$
f_{\lambda_{s}} \geqq\left(\lambda_{1}-\lambda_{s}\right) \cdots\left(\lambda_{k-1}-\lambda_{s}\right)
$$

Proof: As in the proof of Lemma A.2 we have

$$
\begin{aligned}
f_{\lambda_{s}}= & \left(\lambda_{1}-\lambda_{s}\right) f_{\lambda_{s} \lambda_{1}}+f_{\lambda_{1}} \\
\geqq & \left(\lambda_{1}-\lambda_{s}\right) f_{\lambda_{s} \lambda_{1}} \\
= & \left(\lambda_{1}-\lambda_{s}\right)\left(\left(\lambda_{2}-\lambda_{s}\right) f_{\lambda_{s} \lambda_{1} \lambda_{2}}+f_{\lambda_{1} \lambda_{2}}\right) \\
\geqq & \left(\lambda_{1}-\lambda_{s}\right)\left(\lambda_{2}-\lambda_{s}\right) f_{\lambda_{s} \lambda_{1} \lambda_{2}} \\
& \cdots \cdots \cdots\left(\lambda_{k-1}-\lambda_{s}\right) . \\
\geqq & \left(\lambda_{1}-\lambda_{s}\right) \cdots
\end{aligned}
$$

TheOREM A.1. Let $\psi_{0}>0$ be any positive number, $2 \leqq k \leqq n$. We have

$$
\sum_{i} f_{\lambda_{1}} \rightarrow+\infty \text { as }|\lambda| \rightarrow+\infty \text {, }
$$

where $\lambda \in \Gamma, f(\lambda) \geqq \psi_{0}>0$.

Proof: We prove the theorem by a contradiction argument. Assume this is not the case, then along a sequence of $\{\lambda\}$ (we still denote it as $\{\lambda\}$ ), we have

$$
|\lambda| \rightarrow+\infty, f(\lambda) \geqq \psi_{0}>0 \text { and } \sum_{i} f_{\lambda_{i}}(\lambda) \geqq C<+\infty
$$

Let $\lambda_{1} \geqq \cdots \geqq \lambda_{n} ;$ there are two cases:

Case 1. $\lambda_{n} \geqq 0$. In this case, we have

$$
\begin{gathered}
0<\psi_{0} \leqq f(\lambda) \leqq\left(\begin{array}{l}
n \\
k
\end{array}\right) \lambda_{1} \cdots \lambda_{k} \\
f_{\lambda_{n}} \geqq \lambda_{1} \cdots \lambda_{k-1} .
\end{gathered}
$$

It follows that

$$
f_{\lambda_{n}} \geqq \frac{\psi_{0}}{\left(\begin{array}{l}
n \\
k
\end{array}\right)} \frac{1}{\lambda_{k}}
$$


Therefore, $\lambda_{k} \geqq \varepsilon>0$ for some positive number $\varepsilon$. Hence,

$$
f_{\lambda_{n}} \geqq \lambda_{1} \cdots \lambda_{k-1} \geqq \lambda_{1} \varepsilon^{k-2} \rightarrow+\infty \quad \text { as } \quad|\lambda| \rightarrow+\infty
$$

which is a contradiction.

Case 2. There exists some $s \in\{k+1, \cdots, n\}$, such that

$$
\lambda_{1} \geqq \cdots \geqq \lambda_{k} \geqq \cdots \geqq \lambda_{s-1} \geqq 0 \geqq \lambda_{s} \geqq \cdots \geqq \lambda_{n}
$$

From Lemma A.1 we know that $\lambda_{k}>0$. It follows from Lemma A.3 that

$$
\left(\lambda_{1}-\lambda_{n}\right) \cdots\left(\lambda_{k-1}-\lambda_{n}\right) \leqq f_{\lambda_{n}} \leqq \sum_{i} f_{\lambda_{i}} \leqq C
$$

Noticing that $\lambda_{n} \leqq 0$, we have

$$
\lambda_{1} \cdots \lambda_{i}\left|\lambda_{n}\right|^{k-1-i} \leqq C, \quad i=1, \cdots, k-1,
$$

Clearly, $\lambda_{n}$ stays bounded, therefore $\lambda_{1} \rightarrow+\infty$. From (79) we see that

$$
\lambda_{k-1}+\left|\lambda_{n}\right| \rightarrow 0
$$

Thus,

$$
\begin{aligned}
f(\lambda) & \leqq \sum_{i=1}^{k-1} \lambda_{1} \cdots \lambda_{i}\left|\lambda_{n}\right|^{k-i}+\left|\lambda_{n}\right|^{k}+\lambda_{1} \cdots \lambda_{k} \\
& \leqq(k-1) C\left|\lambda_{n}\right|+\left|\lambda_{n}\right|^{k}+C \lambda_{k} \rightarrow 0
\end{aligned}
$$

which is a contradiction.

Lemma A.4. Suppose $\lambda \in \Gamma, n \geqq k \geqq 2$, and

$$
\lambda_{1} \geqq \cdots \geqq \lambda_{n} ;
$$

then, for $l=1, \cdots, n$, we have

$$
f_{\lambda_{1}}(\lambda) \leqq\left(\begin{array}{l}
n-1 \\
k-1
\end{array}\right) \lambda_{1} \cdots \lambda_{k-1}
$$

Proof: $f_{\lambda}$, is a symmetric function of $\lambda_{1}, \cdots, \lambda_{n-1}$ homogeneous of degree $k-1$. The conclusion follows from Lemma A.1.

THEOREM A.2. Suppose $\lambda \in \Gamma$, and

$$
\lambda_{1} \geqq \cdots \geqq \lambda_{n}
$$


There exists some positive number $\delta^{\prime}$, such that, for any $\lambda_{s} \leqq 0, s \in\{1, \cdots, n\}$, we have

$$
f_{\lambda_{s}} \geqq \delta^{\prime} f_{\lambda_{l}}, \quad 1 \leqq l \leqq n .
$$

Proof: According to Lemma A.1, $s>k$. If $k=1$, this is trivial. Let us assume $k \geqq 2$. From Lemma A.3 we know that

$$
f_{\lambda_{s}} \geqq \lambda_{1} \cdots \lambda_{k-1}
$$

Combining the above with Lemma A.4 concludes the proof.

THEOREM A.3. Suppose that $\lambda \in \Gamma$, and

$$
\lambda_{1} \geqq \cdots \geqq \lambda_{n} \geqq 0
$$

Then

$$
f_{\lambda_{1}} \lambda_{1}^{2} \geqq\left(\begin{array}{l}
n \\
k
\end{array}\right)^{-1} \lambda_{1} f(\lambda)
$$

Proof: According to Lemma A.1,

$$
f(\lambda) \leqq\left(\begin{array}{l}
n \\
k
\end{array}\right) \lambda_{1} \cdots \lambda_{k}
$$

On the other hand,

$$
f_{\lambda_{1}} \lambda_{1}^{2} \geqq \lambda_{1}^{2} \lambda_{2} \cdots \lambda_{k}\left(\begin{array}{l}
n \\
k
\end{array}\right)^{-1} \geqq \lambda_{1} f(\lambda)
$$

and the result follows.

Acknowledgment. This is an improvement of part of the author's thesis. The author is greatly indebted to his thesis adviser Professor Nirenberg for leading him to problems of this type and many useful suggestions and comments. He would also like to thank Professor Christodoulou for useful comments. Thanks are also due to Zhengchao Han for helpful discussions.

The work for this paper was partially supported by the Alfred P. Sloan Doctoral Dissertation Fellowship and was completed at Princeton University.

\section{Bibliography}

[1] Agmon, S., Douglis, A., and Nirenberg, L., Estimates near the boundary for solutions of elliptic partial differential equations satisfying general boundany conditions, Comm. Pure. Appl. Math. 12. 1959, pp. 623-727.

[2] Agmon, S., Douglis, A., and Nirenberg, L., Estimates near the boundary for solutions of elliptic partial differential equations satisfying general boundary conditions, Comm. Pure Appl. Math. 17, 1964, pp. 35-92. 
[3] Aubin. T., Nonlinear analysis on manifolds: Monge-Ampère equations, Springer Verlag, 1982.

[4] Caffarelli, L.. Kohn. J. J., Nirenberg, L., and Spruck, J., The Dirichlet problem for nonlinear second-order elliptic equations II: Complex Monge-A mpère and uniformly elliptic equations, Comm. Pure Appl. Math. 38. 1985. pp. 209-252.

[5] Caffarelli, L., Nirenberg. L., and Spruck, J., The Dirichlet problem for nonlinear second-order elliptic equations I: Monge-Ampère equation, Comm. Pure Appl. Math. 37, 1984, pp. 369-402.

[6] Caffarelli, L., Kohn, J. J., and Spruck, J., The Dirichlet problem for nonlinear second-order elliptic equations II: Conplex Monge-Ampère and uniformly elliptic equations, Comm. Pure Appl. Math. 38,1985 , pp. 209-252.

[7] Caffarelli, L., Nirenberg. L., and Spruck, J., The Dirichlet problem for nonlinear second-order elliptic equations III: Functions of the eigenvalues of the Hessian. Acta Math. 155, 1985, pp. $261-301$.

[8] Caffarelli, L.. Nirenberg. L., and Spruck, J., The Dirichlet problem for nonlineur second-order elliptic cquations IV: Starshaped compact Weingarten hypersurfaces, in Current Topics in Partial Differemial Equations, Y. Ohya, K. Kasahara, and N. Shimakura, eds., Kinokunize, Tokyo, 1986, pp. 1-26.

[9] Cheng, S. Y.. and Yau. S. T., Differential equations on Riemannian manifolds and their geometric applications, Comm. Pure Appl. Math. 38, 1975, pp. 333-354.

[10] Evans, L. C.. Classical solutions of fully nonlinear convex second-order elliptic equations, Comm. Pure Appl. Math. 35, 1982, pp. 333-363.

[11] Fitzpatrick, P. M., and Pejsachowicz, J., An extension of the Leray-Schauder degree for fully nonlinear elliptic problems. Symp. in Pure Math., Proc. AMS 45, p. 425.

[12] Gidas, B., Ni, W. M., and Nirenberg, L., Symmetry and related properties via the maximum principle, Comm. Math. Phys. 68, 1979, pp. 209-243.

[13] Gilbarg. D., and Trudinger, N. S., Elliptic Partial-Differential Equations, (2nd ed.), SpringerVerlag. 1983.

[14] Kato. T., Perturhation Theory for Linear Operators, (2nd ed.), Springer-Verlag, 1976.

[15] Korevaar. N. J., An easy proof of the interior gradient bound for solutions to the prescribed mean curvature equations. Proc. of Symp. in Pure Math. 45, 1986.

[16] Korevaar. N. J.. A priori interior gradient bounds for solutions to elliptic Weingarten equations, Ann. Inst. Henri Poincare 4, 1987, pp. 1-17.

[17] Krylov, N. V., Boundedly inhomogeneous ellipuic and parubolic equations in a domain, Izv. Akad. Nauk SSSR 47, 1983, pp. 75-108.

[18] Li, Yan Yan, Degree of second-order fully nonlinear elliptic operators and its applications, to appear.

[19] Serrin, J., A symmetry problem in potential theory, Arch. Rat. Mech. Anal. 43, 1971, pp. 304-318.

Received February, 1989. 